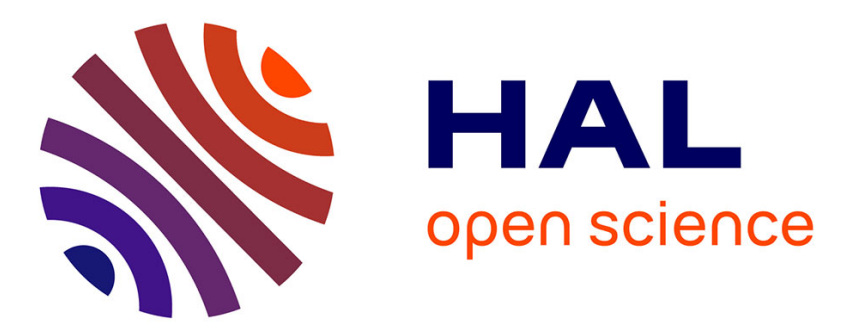

\title{
Cosublimation: A Rapid Route Toward Otherwise Inaccessible Halogen-Bonded Architectures
}

Patrick M.J. Szell, Shaina Gabriel, Estelle Caron-Poulin, Olivier Jeannin, Marc Fourmigué, David Bryce

\section{- To cite this version:}

Patrick M.J. Szell, Shaina Gabriel, Estelle Caron-Poulin, Olivier Jeannin, Marc Fourmigué, et al.. Cosublimation: A Rapid Route Toward Otherwise Inaccessible Halogen-Bonded Architectures. Crystal Growth \& Design, 2018, 18 (10), pp.6227 - 6238. 10.1021/acs.cgd.8b01089 . hal-01894682

HAL Id: hal-01894682

https://hal-univ-rennes1.archives-ouvertes.fr/hal-01894682

Submitted on 15 Oct 2018

HAL is a multi-disciplinary open access archive for the deposit and dissemination of scientific research documents, whether they are published or not. The documents may come from teaching and research institutions in France or abroad, or from public or private research centers.
L'archive ouverte pluridisciplinaire HAL, est destinée au dépôt et à la diffusion de documents scientifiques de niveau recherche, publiés ou non, émanant des établissements d'enseignement et de recherche français ou étrangers, des laboratoires publics ou privés. 


\title{
Cosublimation: A Rapid Route Towards Otherwise Inaccessible
}

\section{Halogen-Bonded Architectures}

\author{
Patrick M.J. Szell ${ }^{\ddagger}$, Shaina A. Gabriel ${ }^{\ddagger}$, Estelle Caron-Poulin ${ }^{\ddagger}$, Olivier Jeannin ${ }^{\dagger}$, Marc \\ Fourmigué $\dagger$, David L. Bryce ${ }^{\dagger *}$ \\ *Author to whom correspondence is to be addressed
}

†Department of Chemistry and Biomolecular Sciences \& Centre for Catalysis Research and Innovation

University of Ottawa

10 Marie Curie Private

Ottawa, Ontario K1N 6N5

Canada

Tel: +1-613-562-5800 ext.2018; fax: +1-613-562-5170

Email: dbryce@uottawa.ca

$\dagger$ Univ Rennes, CNRS, ISCR (Institut des Sciences Chimiques de Rennes) UMR 6226, Campus de Beaulieu 35042, Rennes, France 


\begin{abstract}
Several synthetic techniques are available for the preparation of halogen-bonded adducts, including traditional solvent-evaporation methods and mechanochemistry. With the intention of reducing solvent usage and experimental times, we establish cosublimation as a synthetic technique for preparing halogen-bonded cocrystals, focusing on the $\mathrm{C}-\mathrm{I} \cdots \mathrm{N}$ motif involving nitrogen-containing heterocycles. We have selected four common halogen bond donors $\quad\left(\mathrm{I}_{2}, \quad\right.$ 1,4-diiodobenzene, 1,4-diiodotetrafluorobenzene, 1,3,5-trifluoro-2,4,6triiodobenzene) and four halogen bond acceptors (2,3,5,6-tetramethylpyrazine, 1,4diazabicyclo[2.2.2] octane, 2,2'-bipyridyl, 1-adamantanecarbonitrile), by virtue of their range in volatility, and explored all 16 combinations via the following methods: solvent evaporation, overnight cosublimation in a sealed glass tube using a two-zone furnace, and expedited cosublimation in a vacuum sublimation apparatus. We show that the raw crystals obtained from overnight cosublimation are of suitable quality for single-crystal X-ray diffraction, resulting in five new crystal structures, two of which are, to date, unobtainable through other methods. Of these new structures, the first halogen bond to the nitrile group of 1-adamantanecarbonitrile is reported, resulting in the formation of a supramolecular rotor in a spinning top configuration as evidenced by ${ }^{13} \mathrm{C}$ and ${ }^{15} \mathrm{~N}$ solid-state NMR spectroscopy. Strikingly, the cosublimation approach overcomes an anti-cooperative halogen bonding effect to produce fully saturated cocrystals of the tritopic halogen bond donor 1,3,5-trifluoro-2,4,6-triiodobenzene with 1,4diazabicyclo[2.2.2] octane. Finally, we show that cosublimation can be used to rapidly prepare and purify bulk samples using a vacuum sublimation apparatus, enabling the scalable preparation of halogen-bonded cocrystals within 10 to 30 minutes using common laboratory equipment. The methods described here enable a broad exploration of the polymorphic landscape and may be extended to the facile preparation of other classes of cocrystals.
\end{abstract}




\section{Introduction}

Preparing high-quality crystalline samples suitable for analysis can be a difficult task, and yet, it is paramount to a multitude of chemical and biochemical studies. With the emergence of X-ray crystallography over the last century, several techniques have been proposed for growing crystals, ${ }^{1}$ such as solvent evaporation and vapor diffusion, to name a few. Establishing a range of crystallization techniques is essential for the successful growth of crystals, perhaps even rendering new structures accessible. As a result of various difficulties and particularities encountered in crystal growth, such as twinning, it is often referred to as an art. $^{2}$

Cocrystals, which are composed of two or more molecules often interacting via noncovalent interactions, ${ }^{3,4}$ offer the advantage of increased manipulability and the tailoring desirable physicochemical properties, ${ }^{5,6}$ and are tuneable for various purposes by varying the functional groups on one or more components. ${ }^{7,8}$ The preparation of cocrystals can be challenging, 9,10 in part due to the competition between the cocrystallization and the crystallization of the pure components. However, successfully preparing a cocrystal is often fruitful, and has been a subject of investigation, for instance, in pharmaceuticals ${ }^{11,12,13}$ and optical materials. ${ }^{14,15}$ Moreover, cocrystallization allows for the study of non-covalent interactions, for instance, by investigating their X-ray crystal structures or spectroscopic properties. Indeed, a series of cocrystals can offer insights into non-covalent interactions, which has been particularly advantageous in the field of $\sigma$-hole based interactions, ${ }^{16,17}$ such as halogen bonding.

Halogen bonding $(\mathrm{XB})$ is a non-covalent interaction between the electrophilic region associated with a covalently bonded halogen, ${ }^{18,19}$ named the $\sigma$-hole on the halogen bond donor, ${ }^{20,21}$ and a nucleophile or Lewis base, the halogen bond acceptor. ${ }^{22}$ Recently, halogen 
bonding has experienced a surge of interest, ${ }^{23,24,25,26,27,28}$ in part due to the directionality of the interaction favoring bond angles $\left(\theta_{\mathrm{R}-\mathrm{X} \cdots \mathrm{Y}}\right)$ nearing 180 degrees $^{29}$ and its tuneability, ${ }^{30,31}$ altogether allowing for the rational design of frameworks ${ }^{32,33}$ and molecules relevant to biochemical systems. ${ }^{34,35}$ The presence of a halogen bond can be further rationalized based on the reduced distance parameter, $R_{\mathrm{XB}}$, which is the quotient of the halogen bond distance $\left(d_{\mathrm{X}} \cdots \mathrm{Y}\right)$ and the sum of the van der Waals radii $\left(\Sigma d_{\mathrm{vdw}}\right),{ }^{36}$ given in Eq. 1:

$$
R_{\mathrm{XB}}=\frac{d_{\mathrm{X} \cdots \mathrm{Y}}}{\sum d_{v d w}} \quad \text { Eq. } 1
$$

Halogen bonding has since found prominence in several areas of chemistry, including medicinal chemistry, ${ }^{37,38,39}$ anion recognition, ${ }^{40,41}$ supramolecular rotors, ${ }^{42,43}$ and catalysis, ${ }^{44,45,46}$ to name a few.

Perhaps the most important tool for the investigation of the halogen bond has been single crystal X-ray diffraction (SCXRD), which offers precise atomic coordinates, thereby allowing the halogen bond geometry to be measured. In parallel, solid-state NMR (SSNMR) has been shown to be a powerful complementary crystallographic tool, ${ }^{47,48,49}$ with many recent studies focused on characterizing the halogen bond by observing changes in chemical shifts, ${ }^{50,51,52} \mathrm{~J}$ couplings, ${ }^{53,54}$ and quadrupolar coupling constants. ${ }^{55,56,57}$ We have recently advanced ${ }^{19} \mathrm{~F}$ and ${ }^{13} \mathrm{C}$ SSNMR as probes for the occurrence of halogen bonding to perfluorinated halogen bond donors; ${ }^{58}$ in particular the relevant chemical shifts are sensitive to the local chemical environment. Further, the high sensitivity and the large chemical shift range associated with the ${ }^{19} \mathrm{~F}$ nucleus is ideal for discerning the starting material from the cocrystals, which is also advantageous in identifying new crystal phases. 
Where large quantities of halogen-bonded materials are desired (>100 mg), crystallization methods requiring solvent evaporation or solvent diffusion can be tedious to perform, often requiring many days or perhaps weeks to achieve the desired product in sufficient quantity. Mechanochemistry has been shown to be a favorable route for the halogen bond synthon, ${ }^{54,59,60,61,62}$ offering a scalable and rapid method of preparing halogen-bonded cocrystals, ${ }^{63,64}$ all without the need of large volumes of solvents. However, mechanochemistry by ball milling ${ }^{65}$ has the inconvenience of yielding powdered products, requiring further manipulations in order to prepare a sample for SCXRD.

Sublimation and deposition, which correspond to the phase transitions from solid to gas, and gas to solid, respectively, are fast and scalable methods for crystal growth. ${ }^{66}$ These processes carry several advantages such as simplicity of the experimental apparatus, and the complete absence of solvents. Sublimation performed simultaneously on two compounds has been referred to alternately as codeposition or cosublimation in the literature,${ }^{67,68,69}$ with the technique varying in terms of experimental methodology across studies..$^{70,71,72}$ We proceed with the name cosublimation to emphasize that this process begins with building blocks in their solid state, which are then sublimed in vacuo using a vacuum pump, a heating source, and a surface for the deposition. In the field of halogen bonding, vapor deposition of the donor and acceptor moieties has been shown to form frameworks, but these limited investigations remain focused on surfaces. $^{73}$ For example, the preparation of halogen-bonded cocrystals by physical vapor deposition has been reported on a silicon substrate. ${ }^{74}$ To our knowledge, only a select instance of cosublimation has been reported for the growth of crystals featuring halogen bonds. ${ }^{75}$

Despite this limited exposure, cosublimation does offer several advantages to the field of halogen-bonded cocrystals, including rapid screening capabilities, and the elimination of all 
solvent interactions. Here, we apply cosublimation to a series of halogen bond donors and acceptors, in an effort to uncover structures otherwise inaccessible by solvent-evaporation methods. We investigate the potential of cosublimation for growing single crystals, for preparing bulk quantities of product with a vacuum sublimation apparatus, and the potential of purifying powdered cocrystals by sublimation. In order to grow single crystals suitable for SCXRD, the halogen bond donor and acceptor were sealed in vacuo to opposite ends of a $25 \mathrm{~cm}$ glass tube. Heating the glass tube in a two-zone tube furnace allows for the separate temperature control of the donor and acceptor, favoring the growth of cocrystals by sublimation. To this end, four halogen bond donors and four halogen bond acceptors with a range of volatility have been selected and shown in Figure 1, focusing on the $\mathrm{I} \cdots \mathrm{N}$ motif. All sixteen combinations were explored using two sublimation techniques: using a two-zone tube furnace under static vacuum, and using a sublimation apparatus under dynamic vacuum, henceforth referred to as "overnight cosublimation" and "expedited cosublimation", respectively. The products from both techniques are compared to the products obtained by slow evaporation, and fully analyzed by ${ }^{13} \mathrm{C}$ and ${ }^{19} \mathrm{~F}$ SSNMR, melting point measurements, powder X-ray diffraction (PXRD), infrared (IR) spectroscopy, and elemental analysis.

$\mathrm{I}_{2}$<smiles>Ic1ccc(I)cc1</smiles><smiles>Fc1c(F)c(I)c(F)c(F)c1I</smiles>

3<smiles>Fc1c(I)c(F)c(I)c(I)c1I</smiles><smiles>Cc1nc(C)c(C)nc1C</smiles>

a<smiles>c1ccc(-c2ccccn2)nc1</smiles>

b<smiles>C1=NCCNC1</smiles>

c

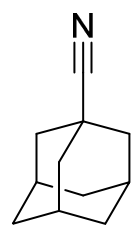

d

Halogen bond donors

Halogen bond acceptors

Figure 1. The pure components of the cocrystals used in this work. The XB donors include: iodine $\left(\mathbf{1}, \mathrm{I}_{2}\right)$, 1,4-diiodobenzene (2, $\left.p \mathrm{DIB}\right)$, 1,4-diiodotetrafluorobenzene $(\mathbf{3}, p \mathrm{DITFB})$, and 1,3,5-trifluoro-2,4,6-triiodobenzene (4, symTFTIB). The XB acceptors include: 2,3,5,6tetramethylpyrazine (a, TMP), 2,2'-bipyridyl (b, BIPY), 1,4-diazabicyclo[2.2.2] octane (c, $\mathrm{DABCO})$, and 1-adamantanecarbonitrile (d, ADCN). 


\section{Experimental}

1,4-diiodobenzene, 2,2'-bipyridyl, 1-adamantanemethanol, (diacetoxyiodo)benzene, 2,2,6,6-tetramethylpiperidine-1-oxyl (TEMPO), 1-adamantanecarbonitrile, and 2,3,5,6tetramethylpyrazine were purchased from Alfa Aesar. Iodine $\left(\mathrm{I}_{2}\right)$ was purchased from Acros Organics. 1,4-diiodotetrafluorobenzene, 1,4-diazabicyclo[2.2.2] octane, and ${ }^{15} \mathrm{~N}$-ammonium acetate $\left(98 \%{ }^{15} \mathrm{~N}\right.$ atoms) were purchased from Sigma Aldrich. 1,3,5-trifluoro-2,4,6triiodobenzene was purchased from Fluorochem. All compounds were used without further purification. All solvents were purchased from a commercial supplier and used without further purification. The ${ }^{15} \mathrm{~N}$-labelled 1-adamantanecarbonitrile was prepared from 1adamantanemethanol and ${ }^{15} \mathrm{~N}$-ammonium acetate using a reported method. ${ }^{76}$ Melting points were measured on an Electrothermal 1A6304 melting point apparatus. The infrared spectroscopy was performed on a Perkin Elmer Spectrum Two ATR FT-IR spectrometer, scanning from $4000 \mathrm{~cm}^{-1}$ to $450 \mathrm{~cm}^{-1}$. The phase purity for each powdered sample was verified by powder X-ray diffraction on a Rigaku Ultima IV instrument with $2 \theta$ ranging from 5 to $55^{\circ}$ in increments of $0.02^{\circ}$ at a rate of $1 \%$ minute using $\mathrm{Cu} \mathrm{K} \alpha$ radiation. No data processing or baseline corrections were employed. All melting points, infrared spectra, powder X-ray diffractograms, elemental analysis, and ${ }^{13} \mathrm{C} /{ }^{19} \mathrm{~F}$ solid-state NMR spectra can be found in the Supporting Information.

Slow evaporation procedure. The halogen bond donor and the halogen bond acceptor were separately dissolved in either acetonitrile, chloroform or ethanol. The halogen bond donor solution was added dropwise to the halogen bond acceptor solution. The solution was left to slowly evaporate over the course of several days, affording the final product. See the Supporting Information for the precise experimental procedures for all 16 combinations. 
Overnight Cosublimation Procedure. To a $25 \mathrm{~cm}$ thin-necked glass tube (see Figure 2a), the halogen bond donor and the halogen bond acceptor were carefully added to opposite ends of the tube. The glass tube was then sealed in vacuo, with a typical pressure of $1 \times 10^{-1} \mathrm{mbar}$. In a home-built, two-zone tube furnace (Figure 2b), both segments of the tube containing the halogen bond donor and acceptor were individually heated from room temperature to an initial temperature of approximately $20^{\circ} \mathrm{C}$ below the melting point, and slowly raised to a temperature of $20{ }^{\circ} \mathrm{C}$ above the melting point, at a rate of $0.04{ }^{\circ} \mathrm{C} /$ minute. The total sublimation times varied, starting from 20 hours, yielding crystals near the center of the tube. The sublimation tube was slowly returned to room temperature over a period of 1 hour, scored with a glass cutting tool, and opened. The crystals were removed and analyzed without further purification. The precise experimental conditions, including pressure, temperatures, and sublimation times for all 16 compounds is given in the Supporting Information.

Expedited Cosublimation Procedure. The halogen bond donor and the halogen bond acceptor were added to a round bottom sublimation apparatus (see Figure 2c). While cooling the cold finger with refrigerated water, the sublimation apparatus was submerged into an oil bath while under a vacuum of $1 \times 10^{-1}$ mbar. The temperature of the oil bath was generally kept between 50 and $100{ }^{\circ} \mathrm{C}$, and varied according to the melting point of the compounds. The sublimation times varied between 10 and 30 minutes, affording the product on the cold finger. See the Supporting Information for the precise experimental conditions for the preparation of all 16 combinations.

${ }^{13}$ C solid-state NMR. All ${ }^{13} \mathrm{C}$ cross-polarization magic-angle spinning (CP/MAS) solid-state NMR experiments were performed at $9.4 \mathrm{~T}\left(v_{\mathrm{L}}\left({ }^{13} \mathrm{C}\right)=100.6 \mathrm{MHz}\right)$ using a Bruker $4 \mathrm{~mm} \mathrm{HXY}$ probe and a Bruker Avance III NMR spectrometer. ${ }^{1} \mathrm{H} \rightarrow{ }^{13} \mathrm{C} \mathrm{CP}$ was used with a $4.6 \mu$ s proton $\pi / 2$ pulse, a $2000 \mu$ s contact time, and a $54.3 \mathrm{kHz}{ }^{1} \mathrm{H}$ decoupling frequency. ${ }^{13} \mathrm{C}$ chemical shifts 
were referenced to glycine at $176.6 \mathrm{ppm}\left({ }^{13} \mathrm{C}=\mathrm{O}\right)$ relative to tetramethylsilane (TMS). A consistent spinning speed of $8 \mathrm{kHz}$ was used for all samples, and 512 transients were acquired for all cocrystals. For the cocrystals, the recycle delay was $3 \mathrm{~s}$ for series $\mathbf{c}(\mathrm{DABCO})$ and $\mathbf{d}$ (ADCN), $5 \mathrm{~s}$ for series a (TMP), and $20 \mathrm{~s}$ for the series b (bipy). Experimental details for the starting materials are given in Table S1 of the Supporting Information.

${ }^{19}$ F solid-state NMR. All ${ }^{19} \mathrm{~F}$ NMR experiments were performed at $7.05 \mathrm{~T}\left(\mathrm{v}_{\mathrm{L}}\left({ }^{19} \mathrm{~F}\right)=282.5\right.$ $\mathrm{MHz}$ ) on a Bruker Avance spectrometer, using a Bruker 2.5 mm HX MAS probe with a spinning frequency of $25 \mathrm{kHz}$. A Hahn echo $(\pi / 2-\tau-\pi-\tau)$ was used with a $\pi / 2$ pulse length of $2.30 \mu \mathrm{s}$, a $\tau$ delay of 2 rotor periods, a recycle delay of $20 \mathrm{~s}$, and 16 transients were collected. The spectra were referenced to $\mathrm{BF}_{3} \cdot \mathrm{OEt}_{2}$ at $-152.8 \mathrm{ppm}$ relative to $\mathrm{CFCl}_{3}{ }^{77}$

Single crystal X-ray diffraction. Single crystals of $\mathbf{2 a}$, 3a-ii, $\mathbf{4 b}$, and $\mathbf{4 c - i i i}$ were mounted on a cryoloop, while a crystal of $\mathbf{3 d}$ was mounted on a thin glass fibre using paraffin oil. The data were collected on a APEX II Bruker AXS Diffractometer at room temperature for 2a, 3a-ii, 4b, 4c-iii, and at 200(2) K for 3d, using graphite-monochromated Mo-K $\alpha$ radiation $(\lambda=0.71073 \AA)$. The raw data collection and reduction were done with the Bruker APEX II software package. ${ }^{78}$ Structures were solved by direct methods (SIR-92) ${ }^{79}$ and refined (SHELXL-2014/7) ${ }^{80}$ by fullmatrix least-squares methods, as implemented in the WinGX software package. ${ }^{81}$ Absorption corrections were applied. Hydrogen atoms were introduced at calculated positions (riding model), included in structure factor calculations, and not refined. Crystallographic data have been deposited with the Cambridge Crystallographic Data Centre as supplementary publication data: CCDC 1854043 for 2a, CCDC 1854044 for 3a-ii, CCDC 1854304 for 3d, CCDC 1854045 for $\mathbf{4 b}$, and CCDC 1854052 for $\mathbf{4 c - i i i . ~ C o p i e s ~ o f ~ t h e ~ d a t a ~ c a n ~ b e ~ o b t a i n e d ~ f r e e ~ o f ~ c h a r g e ~ o n ~}$ application to CCDC, 12 Union Road, Cambridge CB2 1EZ, UK [fax: (+44) 1223-336-033; e- 
mail: deposit@ccdc.cam.ac.uk]. Figures were generated with Diamond version 4.5.1. ${ }^{82}$ Displacement ellipsoid plots were produced using ORTEP, ${ }^{81}$ and uncertainties were estimated using PLATON. ${ }^{83}$

\section{Results and Discussion}

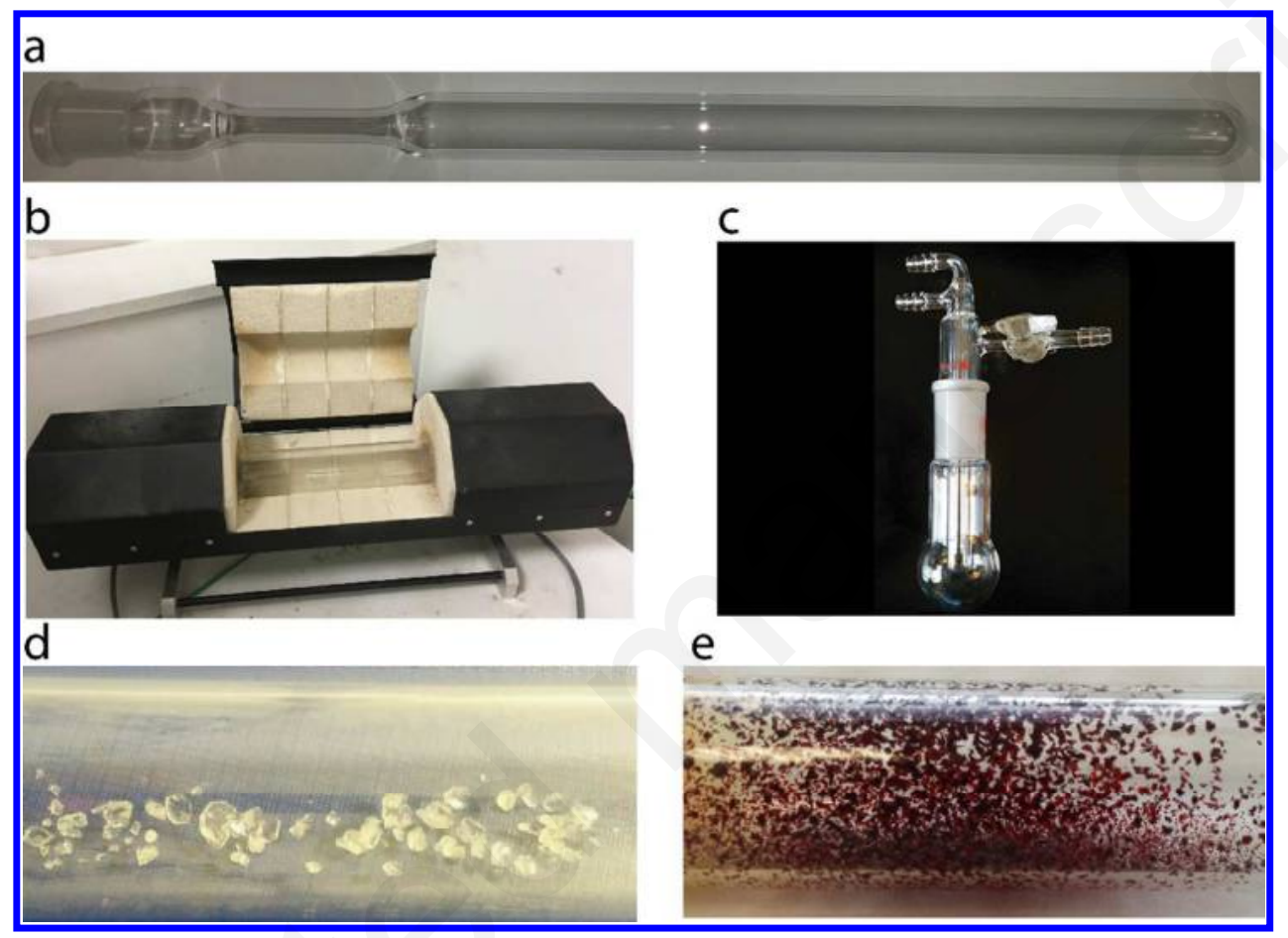

Figure 2. (a) Empty $25 \mathrm{~cm}$ thin-necked glass tube. The halogen bond donor and acceptor are added to opposite extremities of the tube and sealed in vacuo. (b) Two-zone tube furnace used for the overnight cosublimation. (c) Sublimation apparatus used for the expedited cosublimation. Crystals of 3a-ii (d), and 1a (e), obtained by overnight cosublimation.

\section{i-Cocrystal Growth by Overnight Cosublimation}

Cocrystallizations by overnight cosublimation (see Figure 2 (a) and (b)) fared exceptionally well, yielding crystals suitable for SCXRD in almost all cases. Examples of 
crystals obtained from sublimation are shown in Figure 2 (d) and (e). As these trials were attempted at the $300 \mathrm{mg}$ scale in order to isolate sufficient quantities for analysis, sublimation times were between 20 and 72 hours, although sublimation times were shorter when performed on a smaller scale. In contrast, crystal growth by slow evaporation typically required several days, with the total time varying according to the solvent and its evaporation rate. Of the sixteen combinations attempted here, twelve combinations successfully cosublimed, from which five new crystal structures were identified: 2a, 3a-ii, 3d, 4b, and 4c-iii. Notably, structures 3a-ii and $\mathbf{4 c}$-iii were only observed by cosublimation, while structures $\mathbf{3 a - i},{ }^{84} \mathbf{4 c - i},{ }^{85}$ and $\mathbf{4 c - i i}{ }^{\mathbf{8 6}}$ obtained from slow evaporation have been previously described. A summary of the XB geometries and selected crystallographic parameters of all the structures are given in Table 1, and Table 2, respectively. As for the four failed combinations (1d, $\mathbf{2 b}, \mathbf{2 d}$, and $\mathbf{4 d})$, these cocrystals were also not accessible by slow evaporation, with the failure attributed to donor-acceptor incompatibility. For instance, the XB acceptor ADCN is the weakest among the acceptors studied here, and only yielded cocrystals with $p$ DITFB, forming structure 3d. In cases $\mathbf{1 b}$ and $\mathbf{1 c}$, both the PXRD and ${ }^{13} \mathrm{C}$ SSNMR support the occurrence of a mixed phase product, perhaps due to the instability associated with cocrystals featuring $\mathrm{I}_{2}$. A description of the new crystal structures is given below, highlighting their differences with previously reported structures. 
Table 1. Summary of the halogen-bonded cocrystals obtained by cosublimation performed in a glass tube and in a vacuum sublimation apparatus.

\begin{tabular}{lllll}
\hline Acceptor $\backslash$ Donor & $\mathrm{I}_{2}(\mathbf{1})$ & $p$ DIB (2) & $p$ DITFB (3) & symTFTIB (4) \\
\hline TMP (a) & successful & new structure & new structure & successful \\
BIPY (b) & inconclusive & no cocrystals & successful & new structure \\
DABCO (c) & inconclusive & successful & successful & new structure \\
ADCN (d) & no cocrystals & no cocrystals & new structure & no cocrystals \\
\hline
\end{tabular}


Table 2. Summary of the $\mathrm{I} \cdots \mathrm{N}$ halogen bonding geometries observed in this study. The parameters include: the halogen bond length $\left(d_{\mathrm{I} \cdots \mathrm{N}}\right)$, the normalized distance parameter of the halogen bond $\left(R_{\mathrm{XB}}\right)$, the halogen bond angle $\left(\theta_{\mathrm{R}-\mathrm{I} \cdots \mathrm{N}}\right)$, the CSD reference number, and appropriate citation. Experimental errors are given in parentheses.

\begin{tabular}{|c|c|c|c|c|c|}
\hline compound & $\begin{array}{l}\text { XB length } \\
\left(d_{\mathrm{I} \cdots \mathrm{Y}}\right) / \AA\end{array}$ & $R_{\mathrm{XB}}{ }^{\mathrm{a}}$ & $\begin{array}{l}\text { XB angle } \\
\theta_{\mathrm{R}-\mathrm{I} \cdots \mathrm{N}}\left({ }^{\circ}\right)^{b}\end{array}$ & CSD No. & Reference \\
\hline $1 \mathbf{a}$ & $3.086(4)$ & 0.87 & 177.5 & VUKDOU & $\begin{array}{l}\text { R.D. Bailey et } \\
\text { al. }^{75}\end{array}$ \\
\hline $1 b$ & $2.604(1)$ & 0.74 & 179.4 & CECZAL & S. Pohl ${ }^{87}$ \\
\hline 1c & $2.366(4)$ & 0.67 & 178.4 & HEKZEE & $\begin{array}{c}\text { A. Peuronen et } \\
\text { al. }^{88}\end{array}$ \\
\hline 1d & - & - & - & - & no cocrystals \\
\hline $2 a$ & $3.298(5)$ & 0.93 & $167.25(17)$ & - & this work \\
\hline $2 b$ & - & - & - & - & no cocrystals \\
\hline 2c & $\begin{array}{l}2.928(5) \\
2.959(5)\end{array}$ & $\begin{array}{l}0.83 \\
0.84\end{array}$ & $\begin{array}{l}175.4(2) \\
176.3(2)\end{array}$ & VOMHOV & $\begin{array}{c}\text { D. Cinčić et } \\
\text { al. }^{89}\end{array}$ \\
\hline 2d & - & - & - & - & no cocrystals \\
\hline 3a-i & $3.067(2)$ & 0.87 & $177.15(7)$ & JAQMAQ & $\begin{array}{c}\text { J.-L. Syssa- } \\
\text { Magalé et al. }{ }^{84}\end{array}$ \\
\hline 3a-ii & $3.001(4)$ & 0.85 & $177.51(15)$ & - & this work \\
\hline $3 b-i$ & $3.158(7)$ & 0.89 & 169.61(11) & ISIJEZ01 & $\begin{array}{l}\text { J.-L. Syssa- } \\
\text { Magalé et al. }^{84}\end{array}$ \\
\hline 3b-ii & $3.111(3)$ & 0.88 & 169.37(9) & ISIJEZ & M. Bolte \\
\hline $3 c$ & $\begin{array}{l}2.739(5) \\
2.748(5)\end{array}$ & $\begin{array}{l}0.78 \\
0.78\end{array}$ & $\begin{array}{c}173.09(2) \\
174.2(2)\end{array}$ & $\begin{array}{l}\text { ISIHUN01 } \\
\text { ISIHUN02 }\end{array}$ & $\begin{array}{c}\text { Cinčić et al. }{ }^{91} \\
\text { and Pfrunder et } \\
\text { al. }^{86}\end{array}$ \\
\hline 3d & $3.050(4)$ & 0.86 & $173.88(9)$ & & this work \\
\hline $4 a$ & $\begin{array}{l}2.991(3) \\
2.993(3)\end{array}$ & $\begin{array}{l}0.85 \\
0.85\end{array}$ & $\begin{array}{l}178.09(12) \\
179.81(11)\end{array}$ & SAJCUE & $\begin{array}{c}\text { P.M.J. Szell et } \\
\text { al. }^{58}\end{array}$ \\
\hline $4 b$ & $\begin{array}{l}2.968(6) \\
2.969(6)\end{array}$ & $\begin{array}{l}0.84 \\
0.84\end{array}$ & $\begin{array}{l}170.0(2) \\
171.8(2)\end{array}$ & - & this work \\
\hline $4 c-i$ & $2.757(3)$ & 0.78 & $173.89(11)$ & DATCIM & $\begin{array}{l}\text { M.C. Pfrunder } \\
\quad \text { et al. }\end{array}$ \\
\hline $4 c-i i$ & $\begin{array}{l}2.874(4) \\
2.866(4)\end{array}$ & $\begin{array}{l}0.81 \\
0.81\end{array}$ & $\begin{array}{l}166.11(15) \\
172.55(15)\end{array}$ & RORWIG & $\begin{array}{c}\text { J.-L. Syssa- } \\
\text { Magalé et al. }^{86}\end{array}$ \\
\hline 4c-iii & $\begin{array}{l}2.801(4) \\
2.855(3) \\
2.909(4)\end{array}$ & $\begin{array}{l}0.79 \\
0.81 \\
0.82\end{array}$ & $\begin{array}{l}176.56(16) \\
168.80(13) \\
167.73(13)\end{array}$ & - & this work \\
\hline $4 d$ & - & - & - & - & no cocrystals \\
\hline
\end{tabular}




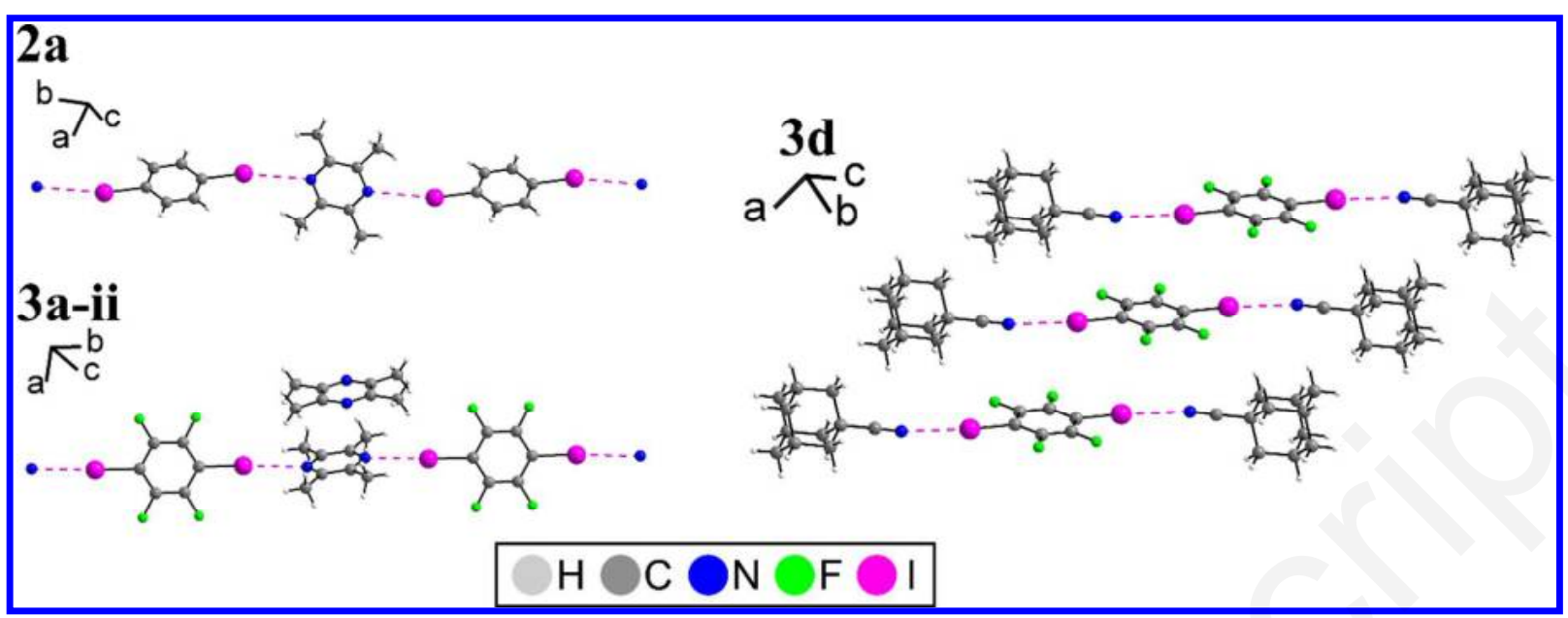

Figure 3. Detailed view of the halogen-bonded networks from the X-ray crystal structures of the cocrystals 2a (upper left), 3a-ii (lower left), and 3d (right) obtained in this study. The dashed magenta line denotes an iodine-nitrogen halogen bond.

Structure 2a shares the same motif as structure $\mathbf{3 a - i},{ }^{84}$ featuring an alternating chain of $\mathbf{2} \cdots \mathbf{a} \cdots \mathbf{2} \cdots \mathbf{a}$, shown in Figure 3. However, the molecular plane of $\mathbf{2}$ is nearly perpendicular to the molecular plane of a, which contrasts with the structure of 3a-i, where the molecular planes of $\mathbf{3}$ and $\mathbf{a}$ are nearly parallel. Furthermore, the XB in structure $\mathbf{2 a}\left(R_{\mathrm{XB}}=0.93, d_{\mathrm{I} \cdots \mathrm{N}}=3.298(5)\right.$ $\left.\AA, \theta_{\mathrm{C}-\mathrm{I} \cdots \mathrm{N}}=167.25(17)^{\circ}\right)$ is much longer and less linear than that in structure 3a-i $\left(R_{\mathrm{XB}}=0.87\right.$, $\left.d_{\mathrm{I} \cdots \mathrm{N}}=3.067(2) \AA, \theta_{\mathrm{C}-\mathrm{I} \cdots \mathrm{N}}=177.15(7)^{\circ}\right)$. Notably, structure 2a has the highest $R_{\mathrm{XB}}$ and therefore the longest XB in this study, seen in Table 2. The longer XB and lower linearity is attributed to the lack of fluorine atoms on $\mathbf{2}$, which would otherwise strengthen the $\mathrm{XB}{ }^{30}$ Interestingly, the transparent crystals of $\mathbf{2 a}$ opacified over the course of a few days, indicating possible metastability. This metastability is further supported by the PXRD and ${ }^{13} \mathrm{C}$ SSNMR spectra (see Figures S10 and S11 of the Supporting Information), showing a lower agreement between the samples obtained from slow evaporation and cosublimation. 
The structure 3a-ii, shown in Figure 3, was only observed by overnight cosublimation, with the PXRD and ${ }^{19} \mathrm{~F}$ SSNMR suggesting an absence of this crystalline phase in the product obtained by slow evaporation (See Figures S16 and S18 of the Supporting Information). The structure 3a-ii is unique from $\mathbf{3 a - i},{ }^{84}$ having a $1: 2$ ratio between $\mathbf{3}$ and $\mathbf{a}$, instead of a $1: 1$ ratio. Overall, the structure features a $3 \cdots \mathbf{a} \cdots \mathbf{3} \cdots \mathbf{a} \mathrm{XB}$ chain, with an extra molecule of a not participating in XB. Rather, the molecules of a interact with each other via $\pi$-stacking, forming columns of $\mathbf{a}$ in alternating right angles. The two unique molecules of a can be discerned in the ${ }^{13} \mathrm{C}$ SSNMR spectra of 3a-ii obtained from overnight cosublimation (see Figure S17 of the Supporting Information), with the chemical shift differences between both molecules attributed to the occurrence of the XB to only one a. In our previous study, ${ }^{58}$ we reported an increase in the ${ }^{13} \mathrm{C}$ chemical shift of the methyl groups of a upon $\mathrm{XB}$, whereas the carbons covalently bonded to the nitrogen decreased in chemical shift. Further, as the XB geometry in 3a-ii $\left(R_{\mathrm{XB}}=0.85, d_{\mathrm{I} \cdots \mathrm{N}}\right.$ $\left.=3.001(4) \AA, \theta_{\mathrm{C}-\mathrm{I} \cdots \mathrm{N}}=177.51(15)^{\circ}\right)$ is subtly shorter than the $\mathrm{XB}$ geometry observed in 3a-i $\left(d_{\mathrm{I} \cdots \mathrm{N}}=3.158(3) \AA\right)$, a lower ${ }^{13} \mathrm{C}$ chemical shift corresponding to the carbons covalently bonded to nitrogen is observed in 3a-ii $(147.2 \pm 0.2 \mathrm{ppm})$ than in $\mathbf{3 a - i}(149.8 \pm 0.3 \mathrm{ppm})$.

Structure 3d, shown in Figure 3, features the first instance of XB to the nitrile group of $\mathrm{ADCN}$ in the Cambridge Structural Database $(\mathrm{CSD}) .^{92}$ The XB geometry $\left(R_{\mathrm{XB}}=0.86, d_{\mathrm{I} \cdots \mathrm{N}}=\right.$ $\left.3.050(4) \AA, \theta_{\mathrm{C}-\mathrm{I} \cdots \mathrm{N}}=173.88(9)^{\circ}\right)$ is moderately short, consistent with the fact that nitrile groups are not the strongest nitrogen-containing XB acceptors. ${ }^{93}$ As a result of the electron donating nature of the adamantyl group, $\mathrm{ADCN}$ is a relatively strong nitrile $\mathrm{XB}$ acceptor. ${ }^{94}$ While the $\mathrm{C} \equiv \mathrm{N} \cdots \mathrm{I}$ bond angles in the $\mathrm{CSD}$ range between 90 and $180^{\circ}$, here, the $\mathrm{XB}$ is coordinating the apex of the nitrile group, resulting in a $\mathrm{C} \equiv \mathrm{N} \cdots \mathrm{I}$ bond angle of $167.43^{\circ}$. 


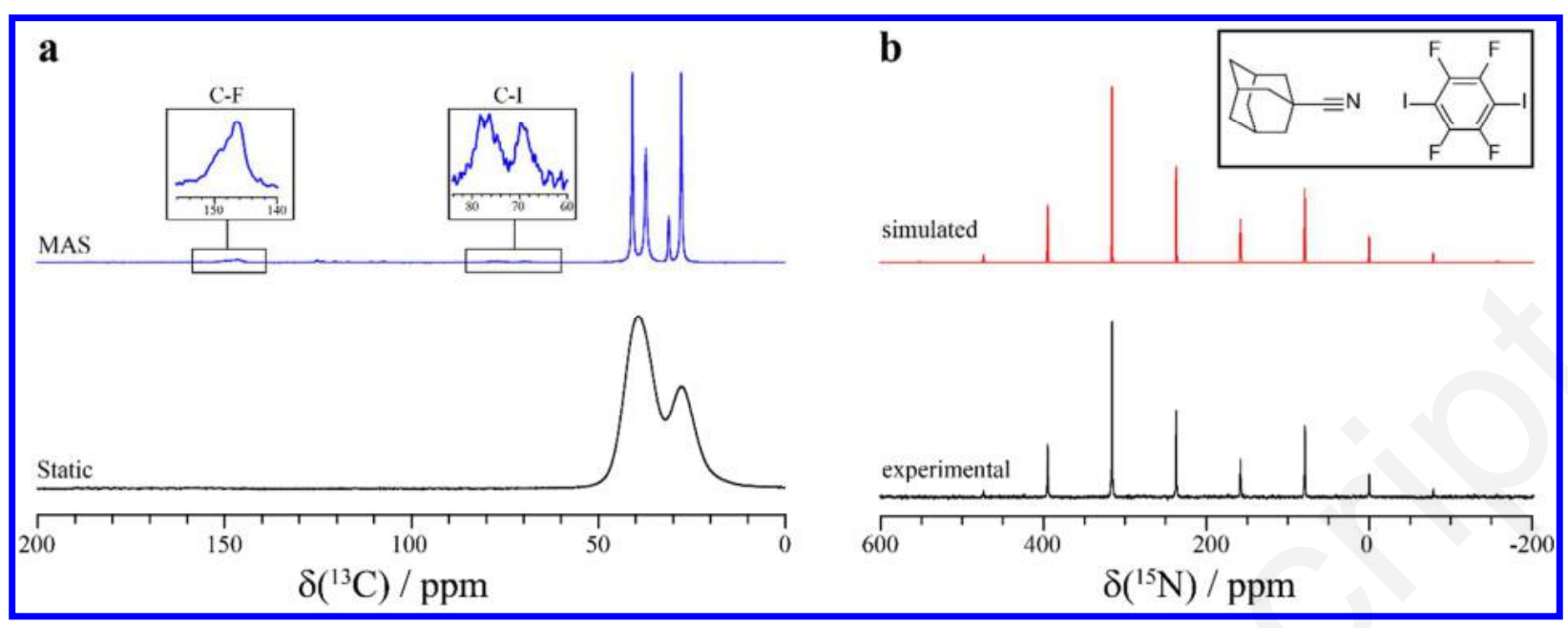

Figure 4. ${ }^{13} \mathrm{C}$ and ${ }^{15} \mathrm{~N}$ (right) solid-state NMR spectra of compound 3d. (a) Experimental ${ }^{13} \mathrm{C}$ SSNMR spectra acquired under static (bottom left, black) and MAS conditions (upper, blue). Magnified views of the C-F and C-I resonances are shown in the insets. (b) Experimental ${ }^{15} \mathrm{~N}$ SSNMR spectrum acquired at a $4 \mathrm{kHz}$ MAS frequency (bottom, black), and the WSOLIDS ${ }^{95}$ simulated spectrum (upper, red).

As molecular adamantane is known for its molecular rotations in the solid state, ADCN has been previously investigated for its molecular rotation. ${ }^{96,97,98}$ It can be seen in the crystal structure of $\mathbf{3 d}$ acquired at 200(2) K that there is a lack of contacts to the adamantane portion of ADCN, suggesting that related molecular rotations may also be present in the cocrystals. This is further supported by the ${ }^{13} \mathrm{C}$ SSNMR spectrum acquired at room temperature under static conditions, which has a relatively narrow line width of $950 \pm 25 \mathrm{~Hz}$ for the $\mathrm{CH}_{2}$ carbons, despite the lack of magic angle spinning. This narrowing of the resonances can be attributed to the rotation of the adamantane ring, which results in averaging of carbon chemical shift anisotropy and some dipolar interactions. In comparison, molecular adamantane, which is known for its 
plasticity, has a linewidth of $270 \pm 10 \mathrm{~Hz}$ under the same conditions, on the same order as this ADCN cocrystal. In contrast, the ${ }^{15} \mathrm{~N}$ SSNMR performed on the ${ }^{15} \mathrm{~N}$-enriched ADCN cocrystal, shown in Figure 4b, reveals a sizeable chemical shift anisotropy $\left(\delta_{\text {iso }}\left({ }^{15} \mathrm{~N}\right)=237.0 \pm 0.1 \mathrm{ppm} ; \Omega\right.$ $=425 \pm 30 \mathrm{ppm} ; \kappa=1.00 \pm 0.2$ ), attributed to the nitrogen remaining fixed in space as a result of XB. The distinctions between the dynamics displayed in the crystal structure and in the SSNMR spectra may be attributed to the temperature difference of nearly $100 \mathrm{~K}$ during their acquisitions. Overall, this configuration resembles a spinning top, akin to similar reports in the literature. ${ }^{42}$ However, a full dynamical analysis of the ADCN rotations is beyond the scope of this study, and has not been investigated further.

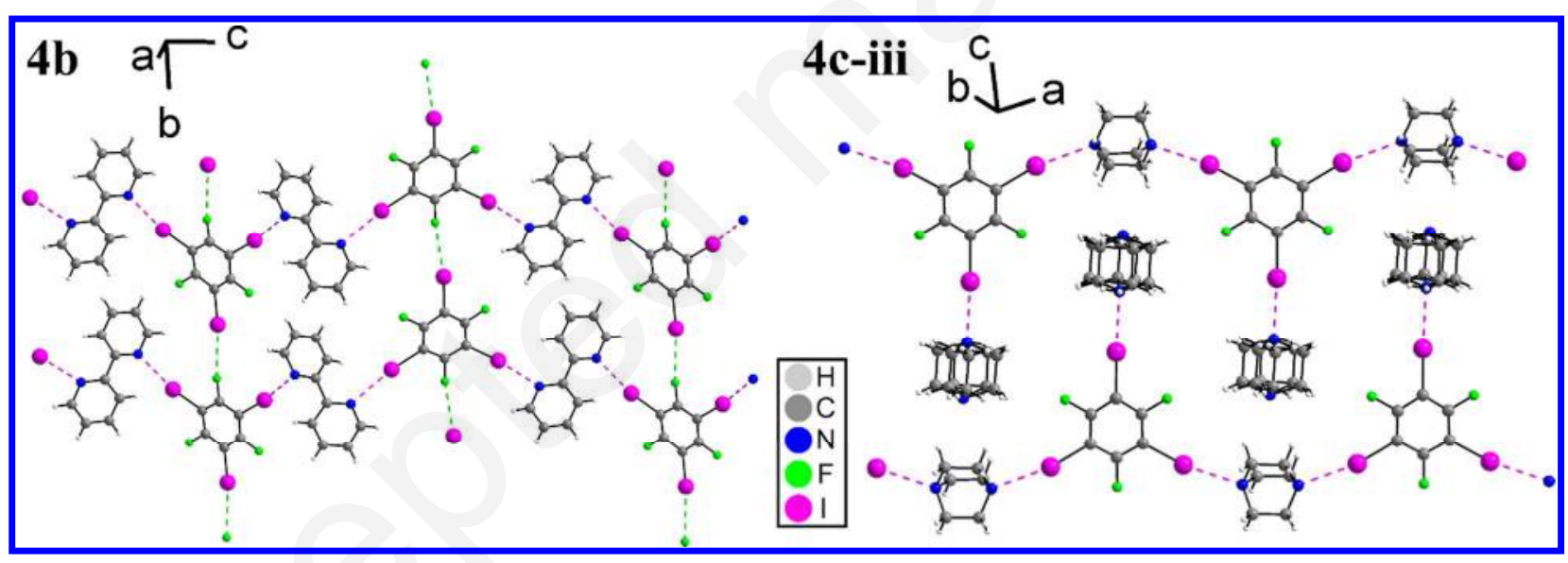

Figure 5. Crystal structures of $\mathbf{4 b}$ (left) and $\mathbf{4 c - i i i}$ (right), obtained by cosublimation.

In structure 4b, shown in Figure 5, BIPY and symTFTIB are cocrystallized in a 1:1 stoichiometry, featuring two nearly equivalent C-I $\cdots \mathrm{N}$ XBs $\left(R_{\mathrm{XB}}=0.84, d_{\mathrm{I} \cdots \mathrm{N}}=2.968(6) \AA, \theta_{\mathrm{C}-}\right.$ $\left.{ }_{\mathrm{I} \cdots \mathrm{N}}=170.0(2)^{\circ} ; R_{\mathrm{XB}}=0.84, d_{\mathrm{I} \cdots \mathrm{N}}=2.969(6), \theta_{\mathrm{C}-\mathrm{I} \cdots \mathrm{N}}=171.8(2)^{\circ}\right) . \quad$ Due to the nitrogen on the 
pyridyl rings being in the trans position, a zig-zag architecture is formed, with the chains linked by a C-I $\cdots \mathrm{F}$ halogen contact $\left(d_{\mathrm{I} \cdots \mathrm{F}}=3.310(4) \AA, \theta_{\mathrm{C}-\mathrm{I} \cdots \mathrm{F}}=172.3(2)^{\circ}\right)$. Structure $4 \mathbf{b}$ is similar to previous reports featuring sym TFTIB and a series of other 4,4'-bipyridyl derivatives, ${ }^{99,100}$ with the resulting donor molecule participating in two instead of the potential three $\mathrm{C}-\mathrm{I} \cdots \mathrm{N}$ XBs. This preference was previously rationalized as an anti-cooperative effect of the XB in polytopic donors, with the C-I $\cdots \mathrm{N}$ binding energy decreasing with each additional XB. ${ }^{99}$ This was further observed in cocrystals featuring $N, N^{\prime}$-diodo-dimethylhydantoin as the $\mathrm{XB}$ donor, with the introduction of a XB affecting the neighboring iodine's capability to form XBs. ${ }^{101}$

Several cocrystal structures featuring symTFTIB and DABCO have been reported, with donor:acceptor stoichiometries varying from $1: 1$ (one C-I $\cdots \mathrm{N} \mathrm{XB})^{85}$ to $1: 2$ (two C-I $\cdots \mathrm{N} \mathrm{XBs).}{ }^{86}$ The structure of $\mathbf{4 c - i i i ~ r e p o r t e d ~ h e r e i n ~ a n d ~ s h o w n ~ i n ~ F i g u r e ~ 5 , ~ h a s ~ a ~ 1 : 2 ~ s t o i c h i o m e t r y ~ b e t w e e n ~}$ the donor and acceptor molecules, and importantly features a full saturation of the donor with all three iodines participating in $\mathrm{C}-\mathrm{I} \cdots \mathrm{N}$ halogen bonds. Notably, this structure has only been observed in the sample obtained from slow overnight cosublimation. Three unique XBs are observed in the structure $\left(R_{\mathrm{XB}}=0.79, d_{\mathrm{I} \cdots \mathrm{N}}=2.801(4) \AA, \theta_{\mathrm{C}-\mathrm{I} \cdots \mathrm{N}}=176.56(16)^{\circ} ; R_{\mathrm{XB}}=0.81, d_{\mathrm{I} \cdots \mathrm{N}}\right.$ $\left.=2.855(3) \AA, \theta_{\mathrm{C}-\mathrm{I} \cdots \mathrm{N}}=168.80(13)^{\circ} ; R_{\mathrm{XB}}=0.82, d_{\mathrm{I} \cdots \mathrm{N}}=2.909(4) \AA, \theta_{\mathrm{C}-\mathrm{I} \cdots \mathrm{N}}=167.73(13)^{\circ}\right)$, resulting in a zig-zag $\mathrm{C}-\mathrm{I} \cdots \mathrm{N}$ backbone complemented by DABCO molecules each participating in one $\mathrm{C}-\mathrm{I} \cdots \mathrm{N} \mathrm{XB}$ (see Figure 5). The large $\sim 0.1 \AA$ distinction between the XB lengths can be rationalized by the anti-cooperativity of the shortest $\mathrm{XB}$, which weakens the next two iodine donor sites. The second shortest XB further weakens the third iodine, resulting in a longer XB. In contrast, despite the different acquisition temperature, the $d_{\mathrm{I} \cdots \mathrm{N}} \mathrm{XB}$ lengths are much shorter in structure 4c-i, which exhibits a single C-I $\cdots \mathrm{N}$ XB $\left(d_{\mathrm{I} \cdots \mathrm{N}}=2.757(3) \AA\right)$, presenting a difference of $0.15 \AA$ in comparison to structure $4 \mathbf{c}$-iii. To our knowledge, only three reports are available in 
the literature featuring a fully-saturated symTFTIB XB donor with neutral XB acceptors, including cocrystals with 4-(N,N-dimethylamino)pyridine, ${ }^{102} 1,3,4$-oxadiazole derivatives, ${ }^{103}$ and a $N$-salicylidene Schiff base. ${ }^{104}$ Shown in Figure $6,{ }^{19}$ F SSNMR was able to rapidly identify new phases in a sample that otherwise includes structures $\mathbf{4 c - i}$ and $\mathbf{4 c - i i i . ~}$

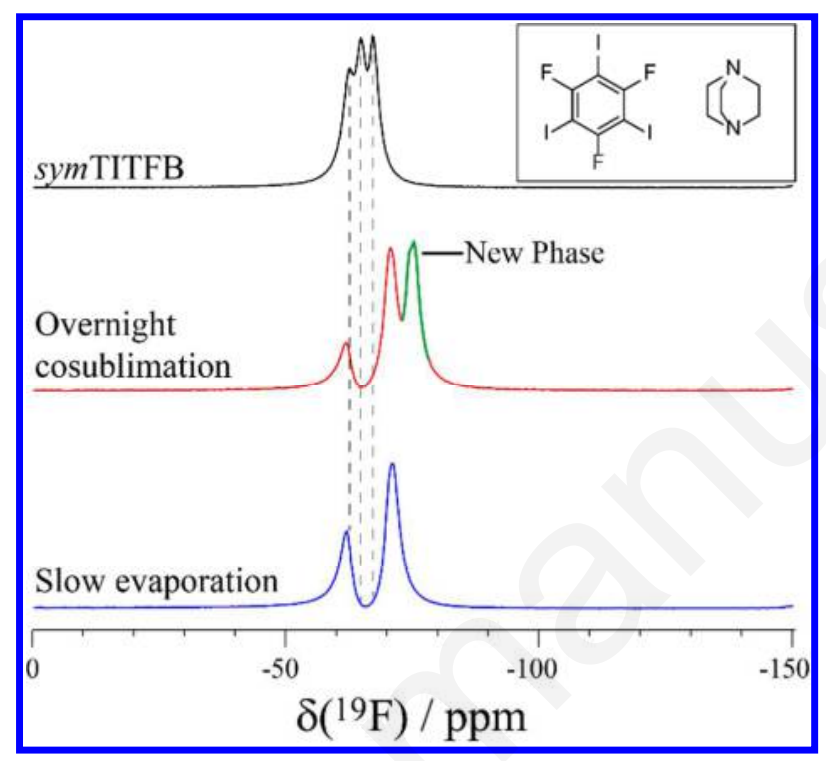

Figure 6. ${ }^{19} \mathrm{~F}$ solid-state NMR $\left(v_{\text {MAS }}=25 \mathrm{kHz}\right)$ spectra of sample $4 \mathbf{c}$ obtained from slow evaporation (blue), overnight cosublimation (red), and the pure starting material (black). The

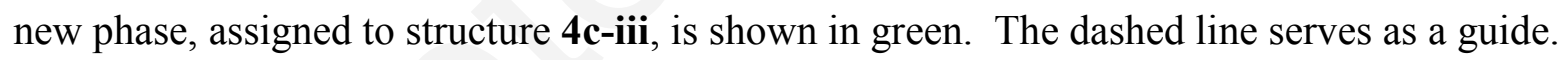

With experimental NMR times of approximately 5 minutes performed on $\sim 12 \mu \mathrm{L}$ of sample and resulting in an excellent signal-to-noise ratio, ${ }^{58}{ }^{19} \mathrm{~F}$ SSNMR of the bulk samples obtained from cosublimation served as a useful tool for quickly identifying new crystallographic phases. An example spectrum of combination $\mathbf{4 c}$ obtained from cosublimation, shown in Figure 6 , is compared to the sample obtained from slow evaporation and the starting material. A new phase can clearly be identified at $-75.3 \pm 0.6 \mathrm{ppm}$; this is assigned to structure $\mathbf{4 c - i i i . ~ T h i s ~ i s ~}$ easily distinguished from the product obtained from slow evaporation, structure $4 \mathbf{c}-\mathbf{i}$, with ${ }^{19} \mathrm{~F}$ 
chemical shifts of $-62.1 \pm 0.3 \mathrm{ppm}$ and $-70.8 \pm 0.3 \mathrm{ppm}$. The lower ${ }^{19} \mathrm{~F}$ chemical shift observed in structure $\mathbf{4 c - i i i}$ is attributed to the presence of three concurrent $\mathrm{XBs}$, with the three fluorine atoms sharing similar chemical environments. However, structure $\mathbf{4 c - i}$ features only one $\mathrm{C}-\mathrm{I} \cdots \mathrm{N}$ XB to DABCO, one C-I $\cdots$ I type II contact, and one I $\cdots$ F contact. Consequently, two fluorine atoms are situated adjacent to the $\mathrm{C}-\mathrm{I} \cdots \mathrm{N}$ XB, while the third fluorine is participating in the $\mathrm{I} \cdots \mathrm{F}$ contact. Due to the nearly 2:1 signal intensity observed for the compound obtained via slow evaporation, the fluorine atom participating in the $\mathrm{I} \cdots \mathrm{F}$ contact has been assigned to the lower chemical shift of $-62.1 \pm 0.3 \mathrm{ppm}$ while the fluorine atoms adjacent to the C-I $\cdots \mathrm{N}$ XB have been assigned to the resonance at $-70.8 \pm 0.3 \mathrm{ppm}$. In general, as a result of the advantages of ${ }^{19} \mathrm{~F}$ SSNMR, it proved to be the most valuable analytical tool in characterizing the fluorinated samples. The general trend observed here is that the ${ }^{19} \mathrm{~F}$ chemical shifts decrease upon C-I $\cdots \mathrm{N}$ halogen bonding, with the resulting spectrum being a rapid and unambiguous indicator of cocrystallization, along with the identification of new crystallographic phases. In contrast, although the ${ }^{13} \mathrm{C}$ chemical shifts of the carbon covalently bonded to nitrogen generally increased upon the formation of a halogen bond, experimental times were significantly longer, required more sample, and the chemical shift changes were small.

The crystals obtained by overnight cosublimation were of diffraction quality, with crystal sizes varying according to sublimation times. In some cases, such as for compound 3a-ii, individual single crystals of up to $85 \mathrm{mg}$ were obtained, which can be useful when large single crystals are required, such as in single crystal NMR. In these cases however, rapid cooling of the crystals should be avoided, as the thermal shock resulted in cracking and popping of larger crystals. However, this was easily avoided by simply allowing the furnace to return to room temperature over a period of $30 \mathrm{~min}$ to $1 \mathrm{~h}$ before removing the sample. Furthermore, of the 
cocrystals studied here, only compound 1c yielded a powder by overnight cosublimation, precluding its analysis by SCXRD. In general, the samples with 1 proved to be the most difficult to prepare by cosublimation, as they were the least consistent with the products obtained by slow evaporation.

In six of the seven successful cocrystals featuring a fluorinated halogen bond donor, the products obtained from overnight cosublimation consisted of a single phase, which was investigated by performing both PXRD and SSNMR on the entirety of the products. This homogeneity was achieved by employing temperature ramps on the order of $0.04{ }^{\circ} \mathrm{C}$ per minute, along with prolonging cosublimation times upon visual inspection. The steady temperature ramps allowed for all starting material to be sublimed and to migrate towards the center of the tube, while avoiding melting. Initial temperatures were chosen to be approximately $20{ }^{\circ} \mathrm{C}$ under the melting point of the compound, and then slowly increased to $20^{\circ} \mathrm{C}$ above the melting point of the compound over the course of several hours. In some cases, the temperatures were adjusted to ensure that the XB donor and acceptor were migrating at equal rates. Delightedly, implementing temperature ramps reduced the need for "finding" precise temperatures, as eventually the compounds would sublime and migrate towards the center of the tube. In the cases of volatile starting materials, such as combinations 1a and 1c, heating was not necessary, and simply sealing the two starting materials in vacuo resulted in the formation of cocrystalline product within minutes. An additional advantage of using cosublimation was the possibility of visually following the cocrystallization in the clear glass tube. The cocrystals prepared here had a different morphology than the starting materials, allowing for the temperatures to be adjusted on the fly, or for the cosublimation times to be extended if required. As a result of the soft and solvent-less nature of cosublimation, the experimental aspects discussed here may be valuable 
for the preparation of cocrystals based on non-covalent interactions other than halogen bonds, such as the emerging chalcogen, pnictogen, and tetrel bonds. ${ }^{17}$ 
Table 3. Selected single-crystal X-ray crystallographic data for compounds $\mathbf{2 a}, \mathbf{3 a - i i}, \mathbf{3 d}, \mathbf{4 b}, \mathbf{4 c}$.

\begin{tabular}{|c|c|c|c|c|c|}
\hline compound & $2 \mathbf{a}$ & 3a-ii & 3d & $4 b$ & 4c-iii \\
\hline \multirow[t]{2}{*}{ empirical formula } & $\mathrm{C}_{14} \mathrm{H}_{16} \mathrm{I}_{2} \mathrm{~N}_{2}$ & $\mathrm{C}_{22} \mathrm{H}_{24} \mathrm{~F}_{4} \mathrm{I}_{2} \mathrm{~N}$ & $\mathrm{C}_{20} \mathrm{H}_{30} \mathrm{~F}_{4} \mathrm{I}_{2} \mathrm{~N}$ & $\mathrm{C}_{16} \mathrm{H}_{8} \mathrm{~F}_{3} \mathrm{I}_{3} \mathrm{~N}_{2}$ & $\mathrm{C}_{18} \mathrm{H}_{24} \mathrm{~F}_{3} \mathrm{I}_{3} \mathrm{~N}$ \\
\hline & & 4 & 2 & & 4 \\
\hline $\mathrm{FW}(\mathrm{g} / \mathrm{mol})$ & 394.37 & 674.25 & 724.34 & 665.94 & 731.11 \\
\hline crystal color & colorless & colorless & colorless & colorless & colorless \\
\hline crystal size $(\mathrm{mm})$ & $\begin{array}{l}0.24 \times 0.23 \\
\times 0.18\end{array}$ & $\begin{array}{l}0.45 \times 0.38 \\
\times 0.09\end{array}$ & $\begin{array}{l}0.507 \times \\
0.252 \times \\
0.129\end{array}$ & $\begin{array}{l}0.27 \times 0.08 \\
\times 0.06\end{array}$ & $\begin{array}{l}0.03 \times 0.02 \\
\times 0.01\end{array}$ \\
\hline crystal system & triclinic & triclinic & monoclinic & $\begin{array}{l}\text { Orthorhomb } \\
\text { ic }\end{array}$ & triclinic \\
\hline crystal space group & $P-1$ & $P-1$ & $P 2{ }_{1} / n$ & $P \cap a 2_{1}$ & $P-1$ \\
\hline$T(\mathrm{~K})$ & $296(2)$ & $296(2)$ & $200(2)$ & $296(2)$ & $296(2)$ \\
\hline$a(\AA)$ & $6.1555(11)$ & $7.3019(11)$ & $6.8334(5)$ & $7.7491(13)$ & $6.5306(5)$ \\
\hline$b(\AA)$ & $6.2811(11)$ & $9.6012(14)$ & $10.4688(8)$ & $9.4426(17)$ & $10.5839(7)$ \\
\hline$c(\AA)$ & $10.4557(19)$ & $10.0739(15)$ & $19.0137(15)$ & $25.814(5)$ & $18.9338(13)$ \\
\hline$\alpha\left(^{\circ}\right)$ & $78.165(6)$ & $69.341(4)$ & 90 & 90 & $80.694(2)$ \\
\hline$\beta\left(^{\circ}\right)$ & $88.432(6)$ & $69.659(4)$ & $96.662(4)$ & 90 & $88.633(2)$ \\
\hline$\gamma\left({ }^{\circ}\right)$ & $85.422(6)$ & $82.976(5)$ & 90 & 90 & $72.192(2)$ \\
\hline$V\left(\AA^{3}\right)$ & $394.37(12)$ & $619.63(16)$ & $1351.01(18)$ & $1888.9(6)$ & $1229.13(15)$ \\
\hline$Z$ & 1 & 1 & 2 & 4 & 2 \\
\hline$R_{l}$ (final) & 0.0431 & 0.0398 & 0.0325 & 0.0257 & 0.0361 \\
\hline$w R_{2}$ (final) & 0.1143 & 0.0791 & 0.0808 & 0.0595 & 0.0672 \\
\hline
\end{tabular}




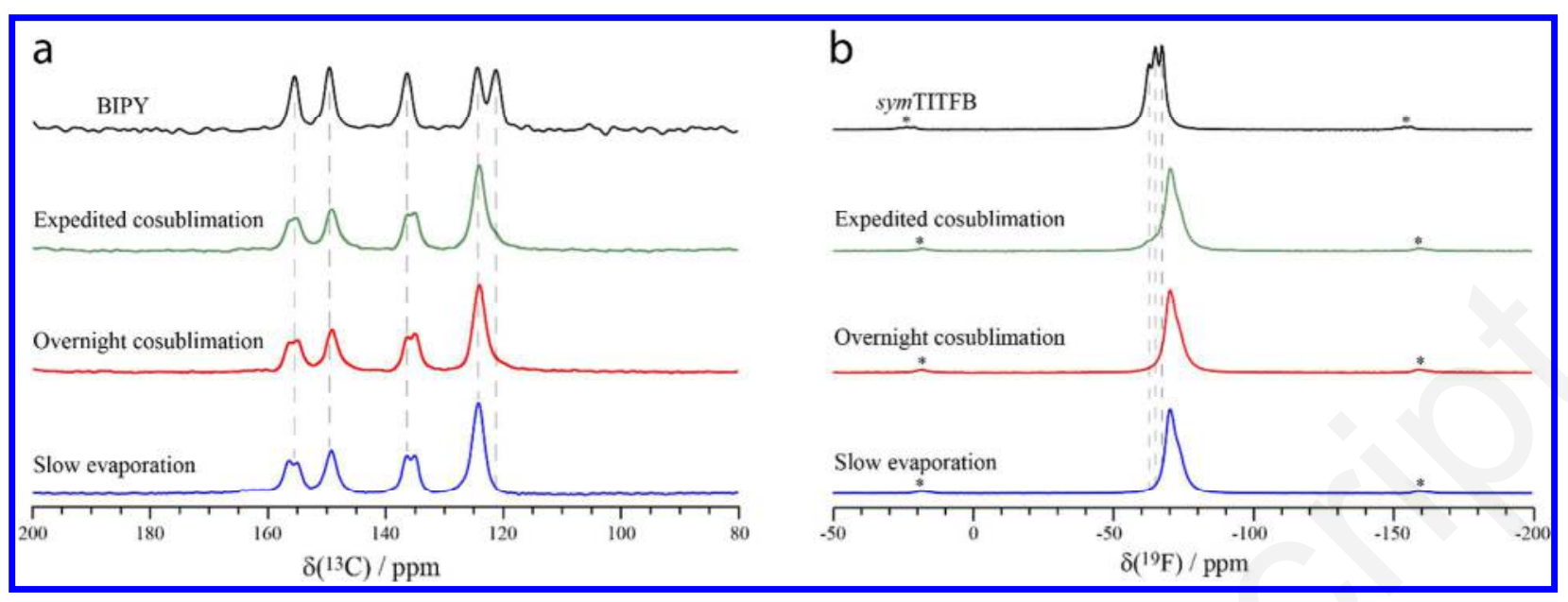

Figure 7. ${ }^{13} \mathrm{C} \mathrm{CP} / \mathrm{MAS}$ (a) and ${ }^{19} \mathrm{~F}$ (b) MAS solid-state NMR spectra of compound $\mathbf{4 b}$ obtained from slow evaporation (blue), overnight cosublimation (red), expedited cosublimation (green), and their corresponding starting materials (black). The dashed lines serve as a guide to discern the starting material from the cocrystals. The asterisks denote spinning sidebands.

\section{ii - Expedited Cosublimation \& Solid State Purification}

The preparation of each cocrystal was attempted by placing both the XB donor and acceptor in a vacuum sublimation apparatus, with this overall process referred to as "expedited cosublimation" in an effort to even further reduce preparation times. The product collected on the cold finger was then compared to the products obtained by slow evaporation and sublimation performed in a glass tube. Expedited cosublimation successfully reproduced the halogen-bonded cocrystals obtained by slow evaporation, with sublimation times ranging between 10 to 30 minutes. As an example, the ${ }^{13} \mathrm{C}$ and ${ }^{19} \mathrm{~F}$ SSNMR spectra of compound $\mathbf{4 b}$ prepared by slow evaporation and both sublimation techniques (in a sealed glass tube and a sublimation apparatus) are shown in Figure 7. The line shapes are superimposable across all synthetic methods, 
indicating that the same product was obtained in each case. Further, apart from preparation 4c, both the PXRD and SSNMR data support the presence of a single phase in the samples successfully prepared by expedited cosublimation. In the case of preparation $\mathbf{4 c}$, a small quantity of a second phase has been observed in the ${ }^{19} \mathrm{~F}$ SSNMR spectrum, assigned to structure $4 \mathbf{c}$-iii. While the sample obtained from the slow evaporation method took several days to prepare, and the sample from overnight cosublimation took approximately $93 \mathrm{~h}$, the sample obtained from the expedited cosublimation apparatus took merely $30 \mathrm{~min}$ with comparable yields, and no solvent usage. Consequently, the shortened preparation times offered by cosublimation can be particularly advantageous for screening a large array of cocrystals.

In contrast to cosublimation performed in a glass tube, single crystals suitable for SCXRD were not obtained when using a vacuum sublimation apparatus. This may be a result of the crystal growth being performed on the order of 30 minutes, resulting in the growth of microcrystalline sample. Furthermore, structure 3c-ii, which was only obtained by overnight cosublimation, was not observed when repeating the procedure in a vacuum sublimation apparatus, perhaps as the technique favors structures with lower conformational energy. As the vacuum sublimation apparatus does not offer the ability to adjust the temperature of the XB donor and acceptor individually, it was more sensitive to vapor pressure mismatch, which was manifested in lower synthetic yields.

In some cases, trace quantities of starting materials were observed in the product obtained from the cold finger on a sublimation apparatus. Separating the starting materials from the cocrystal is challenging, as dissolving the product would sacrifice the cocrystals. As sublimation is traditionally regarded as a purification technique, we attempted to purify the compound by subliming the starting materials, leaving the pure cocrystal. This is rationalized by the 
cocrystallized product having a lower vapor pressure than the starting materials, ${ }^{105}$ with sublimation exploiting this difference. To this end, compound $\mathbf{3 c}$ was prepared by a multitude of synthetic techniques, purposely adding excess donor in some cases, in order to show that sublimation can be relevant to a range of synthetic techniques. These cocrystal preparation methods include: ball milling, ball milling with $20 \%$ excess donor, expedited cosublimation with excess donor and excess heat, liquid assisted grinding in a mortar and pestle with an insufficient grinding time of $1 \mathrm{~min}$, and slow evaporation subsequently doped with $20 \%$ excess donor. Once prepared, each product was split into two parts, with only one part purified by sublimation under a vacuum of approximately $1 \times 10^{-1} \mathrm{mbar}$, a temperature of $70{ }^{\circ} \mathrm{C}$, for up to $15 \mathrm{~min}$. Each product was analyzed by ${ }^{19}$ F SSNMR before and after purification, shown in Figure 8, in order to observe the presence of $p$ DITFB starting material. Additional ${ }^{13}$ C SSNMR, IR spectroscopy, PXRD, and elemental analysis were performed on each product, the results of which can be found in the Supporting Information. 
The impure samples of $\mathbf{3 c}$ clearly show an important presence of excess $p$ DITFB in the ${ }^{19} \mathrm{~F}$ SSNMR spectra, which is denoted by a dashed black line. In contrast, the samples purified by sublimation show a significant reduction in excess starting material, with none being detected in most cases. As for sample 3c prepared by expedited cosublimation, the slight presence of excess $p$ DITFB in the product is attributed to insufficient sublimation times, given the abundance of excess starting material in the impure product. In contrast to the ${ }^{19} \mathrm{~F}$ SSNMR spectra observing the $\mathrm{XB}$ donor, the ${ }^{13} \mathrm{C}$ spectra observing the $\mathrm{XB}$ acceptor were less clear, given the smaller chemical shift changes of $0.4 \pm 0.1 \mathrm{ppm}$ between pure DABCO and the cocrystal. However, the ${ }^{13} \mathrm{C}$ SSNMR spectra support the formation of the cocrystals in each product, with the resulting broader line shape suggesting the presence of excess acceptor in the unpurified products. Upon purification by sublimation, an improvement in the ${ }^{13} \mathrm{C}$ line shapes is observed, 
resulting in superimposable spectra across all samples. Control experiments consisting of heating combination $\mathbf{3 c}$ at atmospheric pressure suggest that the overall purification process is primarily due to sublimation rather than the thermal formation of cocrystals (see Figures S45 and S46 of the Supporting Information). Although heating did result in the formation of cocrystals, it did not remove excess starting materials from the powdered product.

\section{Conclusions}

Cosublimation has allowed for the rapid screening of cocrystals, yielding crystals suitable for single crystal X-ray diffraction, in as little as $20 \mathrm{~h}$. This method has yielded novel and previously inaccessible halogen-bonded architectures. Among the new structural motifs investigated here, a distinguishably weaker and less linear halogen bond is present in structure 2a, in comparison to its perfluorinated analogue, 3a-i. The cosublimation method enabled the preparation of the first cocrystal featuring a halogen bond to the nitrile group of 1adamantanecarbonitrile, resulting in a novel solid-state molecular rotor. Thirdly, the structure of the cocrystal formed between the tritopic halogen bond donor symTFTIB and DABCO, observed only in the sublimated product, reveals a donor fully saturated by halogen bonds, thereby overcoming an anti-cooperative halogen bonding effect. When performed in a vacuum sublimation apparatus, cocrystal preparation times were reduced to under 30 minutes. This technique also provides the opportunity to purify powdered cocrystals. Overall, we have shown that sublimation is a promising technique in the areas of cocrystal engineering and halogen bonding, and has performed exceptionally well for the iconic perfluorinated halogen bond donors. As a result of the forgiving nature of the technique to differences in volatility, it is 
anticipated that cosublimation may find broad usage across the field of crystal engineering and in the study of non-covalent interactions. The method enables broader explorations of the polymorphic energy landscape.

\section{Supporting Information}

The Supporting Information is available free of charge on the ACS Publications website at DOI: 10.1021/xxxxx. Synthetic details, experimental procedures, NMR spectra, IR spectra, PXRD data, ORTEP plots, and elemental analyses are provided.

\section{Conflicts of Interest}

There are no conflicts to declare.

\section{Acknowledgements}

PMJS and DLB thank the Natural Sciences and Engineering Research Council of Canada for a scholarship and for research funding, respectively. We are grateful to Laurie-Anne Roy for technical assistance, and Dr. Bulat Gabidullin for X-ray services.

\section{References}

${ }^{1}$ Nishinaga, T. Progress in art and science of crystal growth and its impacts on modern society. Jpn. J. Appl. Phys. 2015, 54, 050101. 
${ }^{2}$ Gilman, J.J. The Art and Science of Growing Crystals. John Wiley \& Sons Inc. New York, 1963.

3 Aitipamula, S., Banerjee, R.; Bansal, A.K.; Biradha, K.; Cheney, M.L.; Choudhury, A.R.; Desiraju, G.R.; Dikundwar, A.G.; Dubey, R.; Duggirala, N.; Ghogale, P.P.; Ghosh, S.; Goswami, P.K.; Goud, N.R.; Jetti, R.R.K.R.; Karpinski, P.; Kaushik, P.; Kumar, D.; Kumar, V.; Moulton, B.; Mukherjee, A.; Mukherjee, G.; Myerson, A.S.; Puri, V.; Ramanan, A.; Rajamannar, T.; Reddy, C.M.; Rodriguez-Hornedo, N.; Rogers, R.D.; Guru Row, T.N.; Sanphui, P.; Shan, N.; Shete, G.; Singh, A.; Sun, C.C.; Swift, J.A.; Thaimattam, R.; Thakur, T.S.; Thaper, R.K., Thomas, S.P.; Tothadi, S.; Vangala, V.R.; Variankaval, N.; Vishweshwar, P.; Weyna, D.R.; Zaworotko, M.J. Polymorphs, Salts, and Cocrystals: What's in a Name? Cryst. Growth Des. 2012, 12, 2147-2152.

${ }^{4}$ Grothe, E.; Meekes, H.; Vlieg, E.; ter Horst, J.H.; de Gelder, R. Solvates, Salts, and Cocrystals: A Proposal for a Feasible Classification System. Cryst. Growth Des. 2016, 16, 3237-3243.

${ }^{5}$ Bolton, O.; Matzger, A.J. Improved Stability and Smart-Material Functionality Realized in an Energetic Cocrystal. Angew. Chem. Int. Ed. 2011, 50, 8960-8963.

6 Rybtchinski, B. Adaptive Supramolecular Nanomaterials Based on Strong Noncovalent Interactions. ACS Nano. 2011, 5, 6791-6818.

${ }^{7}$ Liu, K.; Kang, Y.; Wang, Z.; Zhang, X. Reversible and Adaptive Functional Supramolecular Materials: "Noncovalent Interaction" Matters. Adv. Mater. 2013, 25, 5530-5548.

${ }^{8}$ Rupasinghe, T.P.; Hutchins, K.M.; Bandaranayake, B.S.; Ghorai, S.; Karunatilake, C.; Bučar, D.-K.; Swenson, D.C.; Arnold, M.A.; MacGillivray, L.R.; Tivanski, A.V. Mechanical Properties of a Series of Macro- and Nanodimensional Organic Cocrystals Correlate with Atomic Polarizability. J. Am. Chem. Soc. 2015, 137, 12768-12771.

${ }^{9}$ Spitzer, D.; Risse, B.; Schnell, F.; Pichot, V.; Klaumünzer, M.; Schaefer, M.R. Continuous engineering of nano-cocrystals for medical and energetic applications. Sci. Rep. 2014, 4, 6575 .

10 Halasz, I.; Puškarić, A.; Kimber, S.A.J.; Beldon, P.J.; Belenguer, A.M.; Adams, F.; Honkimäki, V.; Dinnerbier, R.E.; Patel, B.; Jones, W.; Štrukil, V.; Friščić, T. Real-Time In Situ Powder X-ray Diffraction Monitoring of Mechanochemical Synthesis of Pharmaceutical Cocrystals. Angew. Chem. Int. Ed. 2013, 52, 11538-11541. 
11 Qiao, N.; Li, M.; Schlindwein, W.; Malek, N.; Davies, A.; Trappitt, G. Pharmaceutical cocrystals: An Overview. Int. J. Pharm. 2011, 419, 1-11.

12 Schultheiss, N.; Newman, A. Pharmaceutical Cocrystals and Their Physicochemical Properties. Cryst. Growth Des. 2009, 9, 2950-2967.

${ }^{13}$ Remenar, J.F.; Morissette, S.L.; Peterson, M.L.; Moulton, B.; MacPhee, J.M.; Guzmán, H.R.; Almarsson, Ö. Crystal Engineering of Novel Cocrystals of a Triazole Drug with 1,4Dicarboxylic Acids. J. Am. Chem. Soc. 2003, 125, 8456-8457.

${ }^{14}$ Sun, L.; Zhu, W.; Yang, F.; Li, B.; Ren, X.; Zhang, X.; Hu, W. Molecular cocrystals: design, charge-transfer and optoelectronic functionality. Phys. Chem. Chem. Phys. 2018, 20, 60096023.

15 Christopherson, J.-C.; Topić, F.; Barrett, C.J.; Friščić, T. Halogen-Bonded Cocrystals as Optical Materials: Next-Generation Control over Light-Matter Interactions. Cryst. Growth Des. 2008, 18, 1245-1259.

16 Politzer, P.; Murray, J.S.; Clark, T.; Resnati, G. The $\sigma$-hole revisited. Phys. Chem. Chem. Phys. 2017, 19, 32166-32178.

${ }^{17}$ Cavallo, G.; Metrangolo, P.; Pilati, T.; Resnati, G.; Terraneo, G. Naming Interactions from the Electrophilic Site. Cryst. Growth Des. 2014, 14, 2697-2702.

18 Metrangolo, P.; Meyer, F.; Pilati, T.; Resnati, G.; Terraneo, G. Halogen Bonding in Supramolecular Chemistry. Angew. Chem. Int. Ed. 2008, 47, 6114-6127.

19 Politzer, P.; Murray, J.S.; Clark, T. Halogen Bonding: an electrostatically-driven highly directional noncovalent interaction. Phys. Chem. Chem. Phys. 2010, 12, 7748-7757.

${ }^{20}$ Clark, T.; Hennemann, M.; Murray, J.S.; Politzer, P. Halogen bonding: the $\sigma$-hole. J. Mol. Model. 2007, 13, 291-296.

21 Politzer, P.; Murray, J.S. $\sigma$-Hole Interactions: Perspectives and Misconceptions. Crystals. 2017, 7, 212-226.

${ }^{22}$ Desiraju, G.R.; Ho, P.S.; Kloo, L.; Legon, A.C.; Marquardt, R.; Metrangolo, P.; Politzer, P.; Resnati, G.; Rissanen, K. Definition of the halogen bond (IUPAC Recommendations 2013). Pure Appl. Chem. 2013, 85, 1711-1713.

${ }^{23}$ Cavallo, G.; Metrangolo, P.; Milani, R.; Pilati, T.; Priimagi, A.; Resnati, G.; Terraneo, G. The Halogen Bond. Chem. Rev. 2016, 116, 2478-2601. 
24 Gilday, L.C.; Robinson, S.W.; Barendt, T.A.; Langton, M.J.; Mullaney, B.R.; Beer, P.D. Halogen Bonding in Supramolecular Chemistry. Chem. Rev. 2015, 115, 7118-7195.

${ }^{25}$ Li, B.; Zang, S.-Q.; Wang, L.-Y.; Mak, T.C.W. Halogen Bonding: A powerful, emerging tool for constructing high-dimensional metal-containing supramolecular networks. Coord. Chem. Rev. 2016, 308, 1-21.

26 Berger, G.; Soubhye, J.; Meyer, F. Halogen bonding in polymer science: from crystal engineering to functional supramolecular polymers and materials. Polym. Chem. 2015, 6, 3559-3580.

${ }^{27}$ Lieffrig, J.; Jeannin, O.; Fourmigué, M. Expanded Halogen-Bonded Anion Organic Networks with Star-Shaped Iodoethynyl-Substituted Molecules: From Corrugated 2D Hexagonal Lattices to Pyrite-Type 2-Fold Interpenetrated Cubic Lattices. J. Am. Chem. Soc. 2013, 135, 6200-6210.

28 Riel, A.M.S.; Decato, D.A.; Sun, J.; Massena, C.J.; Jessop, M.J.; Berryman, O.B. The intramolecular hydrogen bonded-halogen bond: a new strategy for preorganization and enhanced binding. Chem. Sci. 2018, 9, 5828-5836.

${ }^{29}$ Huber, S.M.; Scanlon, J.D.; Jimenez-Izal, E.; Ugalde, J.M.; Infante, I. On the directionality of halogen bonding. Phys. Chem. Chem. Phys. 2013, 15, 10350-10357.

${ }^{30}$ Riley, K.E.; Murray, J.S.; Franfrlík, J.; Řezáč, J.; Solá, R.J.; Concha, M.C.; Ramos, F.M.; Politzer, P. Halogen bond tunability I : the effects of aromatic fluorine substitution on the strengths of halogen-bonding interactions involving chlorine, bromine, and iodine. J. Mol. Model, 2011, 17, 3309-3318.

31 Riley, K.E.; Murray, J.S.; Franfrlík, J.; Řezáč, J.; Solá, R.J.; Concha, M.C.; Ramos, F.M.; Politzer, P. Halogen bond tunability II : the varying roles of electrostatic and dispersion contributions to attraction in halogen bonds. J. Mol. Model, 2013, 19, 4651-4659.

32 Priimagi, A.; Cavallo, G.; Metrangolo, P.; Resnati, G. The Halogen Bond in the Design of Functional Supramolecular Materials: Recent Advances. Acc. Chem. Res. 2013, 46, 26862695.

33 Mukherjee, A.; Tothadi, S.; Desiraju, G.R. Halogen Bonds in Crystal Engineering: Like Hydrogen Bonds yet Different. Acc. Chem. Res. 2014, 47, 2514-2524. 
${ }^{34}$ Auffinger, P.; Hays, F.A.; Westhof, E.; Ho, P.S. Halogen bonds in biological molecules. PNAS 2004, 48, 16789-16794.

${ }^{35}$ Scholfield, M.R.; Vander Zanden, C.M.; Carter, M.; Ho, P.S. Halogen bonding (X-bonding): A biological perspective. Protein Sci. 2013, 22, 139-152.

${ }^{36}$ Bondi, A. van der Waals Volumes and Radii. J. Phys. Chem. 1964, 68, 441-451.

${ }^{37}$ Ho, P.S. Halogen bonding in medicinal chemistry: from observation to prediction. Future Med. Chem. 2017, 9, 637-640.

${ }^{38}$ Wilcken, R.; Zimmermann, M.O.; Lange, A.; Joerger, A.C.; Boeckler, F.M. Principles and Applications of Halogen Bonding in Medicinal Chemistry and Chemical Biology. J. Med. Chem. 2013, 56, 1363-1388.

${ }^{39}$ Ford, M.C.; Ho, P.S. Computational Tools To Model Halogen Bonds in Medicinal Chemistry. J. Med. Chem. 2016, 59, 1655-1670.

${ }^{40}$ Ravi, A.; Oshchepkov, A.S.; German, K.E.; Kirakosyan, G.A.; Safonov, Khrustalev, V.N.; Kataev, E.A. Finding a receptor design for selective recognition of perrhenate and pertechnetate: hydrogen $v s$. halogen bonding. Chem. Commun. 2018, 54, 4826-4829.

${ }^{41}$ Molina, P.; Zepata, F.; Caballero, A. Anion Recognition Strategies Based on Combined Noncovalent Interactions. Chem. Rev. 2017, 117, 9907-9972.

${ }^{42}$ Catalano, L.; Perez-Estrada, S.; Wang, H.-H.; Ayitou, A.J.-L.; Khan, S.I.; Terraneo, G.; Metrangolo, P.; Brown, S.; Garcia-Garibay, M.A. Rotational Dynamics of Diazabicyclo[2.2.2]octane in Isomorphous Halogen-Bonded Co-crystals: Entropic and Enthalpic Effects. J. Am. Chem. Soc. 2017, 139, 843-848.

${ }^{43}$ Lemouchi, C.; Vogelsberg, C.S.; Zorina, L.; Simonov, S.; Batail, P.; Brown, S.; GarciaGaribay, M.A. Ultra-fast Rotors for Molecular Machines and Functional Materials via Halogen Bonding: Crystals of 1,4-Bis(iodoethynyl)bicycle[2.2.2]octane with Distinct Gigahertz Rotation at Two Sites. J. Am. Chem. Soc. 2011, 133, 6371-6379.

${ }^{44}$ Chan, Y.-C.; Yeung, Y.-Y. Halogen Bond Catalyzed Bromocarbocyclization. Angew. Chem. Int. Ed. 2018, 57, 3483-3487.

${ }^{45}$ Carreras, L.; Serrano-Torné, M.; van Leeuwen, P.W.N.M.; Vidal-Ferran, A. XBPhos-Rh: a halogen-bond assembled supramolecular catalyst. Chem. Sci. 2018, 9, 3644-3648. 
${ }^{46}$ Gliese, J.-P.; Jungbauer, S.H.; Huber, S.M. A halogen-bonding-catalyzed Michael addition reaction. Chem. Commun. 2017, 53, 12052-12055.

${ }^{47}$ Cerreia Vioglio, P.; Chierotti, M.R.; Gobetto, R. Solid-state nuclear magnetic resonance as a tool for investigating the halogen bond. CrystEngComm. 2016, 18, 9173-9184.

${ }^{48}$ Szell, P.M.J.; Bryce, D.L. Solid-State NMR Studies of Halogen Bonding. Modern Magnetic Resonance, 2016, pp. 1-18. New York: Springer.

${ }^{49}$ Bryce, D.L.; Viger-Gravel, J. Solid-State NMR Study of Halogen-Bonded Adducts. Top. Curr. Chem. 2015, 358, 183-204.

50 Szell, P.M.J.; Cavallo, G.; Terraneo, G.; Metrangolo, P.; Gabidullin, B.; Bryce, D.L. Comparing the Halogen Bond to the Hydrogen Bond by Solid-State NMR: Anion Coordinated Dimers from 2- \& 3-Iodoethynylpyridine Salts. Chem. Eur. J. In press.

${ }^{51}$ Viger-Gravel, J.; Leclerc, S.; Korobkov, I.; Bryce, D.L. Correlation between ${ }^{13} \mathrm{C}$ chemical shifts and the halogen bonding environment in a series of solid paradiiodotetrafluorobenzene complexes. CrystEngComm. 2013, 15, 3168-3177.

52 Cerreia Vioglio, P.; Catalano, L.; Vasylyeva, V.; Nervi, C.; Chierotti, M.R.; Resnati, G.; Gobetto, R.; Metrangolo, P. Natural Abundance ${ }^{15} \mathrm{~N}$ and ${ }^{13} \mathrm{C}$ Solid-State NMR Chemical Shifts: High Sensitivity Probes of the Halogen Bond Geometry. Chem. Eur. J. 2016, 22, 16819-16828.

53 Viger-Gravel, J.; Meyer, J.E.; Korobkov, I.; Bryce, D.L. Probing halogen bonds with solidstate NMR spectroscopy: observation and interpretation of $J\left({ }^{77} \mathrm{Se},{ }^{31} \mathrm{P}\right)$ coupling in halogen-bonded $\mathrm{P}=\mathrm{Se} \cdots$ I motifs. CrystEngComm. 2014, 16, 7285-7297.

${ }^{54}$ Xu, Y.; Viger-Gravel, J.; Korobkov, I.; Bryce, D.L. Mechanochemical Production of HalogenBonded Solids Featuring $\mathrm{P}=\mathrm{O} \cdots \mathrm{I}-\mathrm{C}$ Motifs and Characterization via X-ray Diffraction, Solid-State Multinuclear Magnetic Resonance, and Density Functional Theory. J. Phys. Chem. C 2015, 119, 27104-27117.

55 Szell, P.M.J.; Bryce, D.L. ${ }^{35} \mathrm{Cl}$ Solid-State NMR and Computational Study of Chlorine Halogen Bond Donors in Single-Component Crystalline Chloronitriles. J. Phys. Chem. C 2016, 120, 11121-11130. 
${ }^{56}$ Cerreia Vioglio, P.; Szell, P.M.J.; Chierotti, M.R.; Gobetto, R.; Bryce, D.L. ${ }^{79 / 81}$ Br nuclear quadrupole resonance spectroscopic characterization of halogen bonds in supramolecular assemblies. Chem. Sci. 2018, 9, 4555-4561.

${ }^{57}$ Viger-Gravel, J.; Leclerc, S.; Korobkov, I.; Bryce, D.L. Direct Investigation of Halogen Bonds by Solid-State Multinuclear Magnetic Resonance Spectroscopy and Molecular Orbital Analysis. J. Am. Chem. Soc. 2014, 136, 6929-6942.

${ }^{58}$ Szell, P.M.J.; Gabriel, S.A.; Gill, R.D.D.; Wan, S.Y.H.; Gabidullin, B.; Bryce, D.L. ${ }^{13}$ C and ${ }^{19} \mathrm{~F}$ solid-state NMR and X-ray crystallographic study of halogen-bonded frameworks featuring nitrogen-containing heterocycles. Acta Cryst. 2017, C73, 157-167.

59 Szell, P.M.J.; Dragon, J.; Zablotny, S.; Harrigan, S.R.; Gabidullin, B.; Bryce, D.L. Mechanochemistry and cocrystallization of 3-iodoethynylbenzoic acid with nitrogencontaining heterocycles: concurrent halogen and hydrogen bonding. New J. Chem. 2018, 42, 10493-10501.

${ }^{60}$ Cinčić, D.; Friščić, T.; Jones, W. A Stepwise Mechanism for the Mechanochemical Synthesis of Halogen-Bonded Cocrystal Architectures. J. Am. Chem. Soc. 2008, 130, 7524-7525.

${ }^{61}$ Choquesillo-Lazarte, D.; Nemec, V.; Cinčić, D. CrystEngComm. 2017, 19, 5293-5299.

${ }^{62}$ Mavračić, J.; Cinčić, D.; Kaitner, B. CrystEngComm. 2016, 18, 3343-3346.

${ }^{63}$ Eraković, M.; Nemec, V.; Lež, T.; Porupski, I.; Stilinović, V.; Cinčić, D. Halogen Bonding of $N$-Bromophthalimide by Grinding and Solution Crystallization. Cryst. Growth Des. 2018, $18,1182-1190$.

${ }^{64}$ Lisac, K.; Nemec, V.; Topić, F.; Arhangelskis, M.; Hindle, P.; Tran, R.; Huskić, I.; Morris, A.J.; Friščić, T.; Cinčić, D. Experimental and Theoretical Investigation of Structures, Stoichiometric Diversity, and Bench Stability of Cocrystals with a Volatile Halogen Bond Donor. Cryst. Growth Des. 2018, 18, 2387-2396.

${ }^{65}$ Katsnis, A.D.; Puškarić, A.; Štrukil, V.; Mottillo, C.; Julien, P.A.; Užarević, K.; Pham, M.-H.; Do, T.-D.; Kimber, S.A.J.; Lazić, P.; Magdysyuk, O.; Dinnebier, R.E.; Halasz, I.; Friščić, T. In Situ X-ray diffraction monitoring of a mechanochemical reaction reveals a unique topology metal-organic framework. Nat. Comm. 2015, 6, 6662.

${ }^{66}$ Mullin, J.W. Crystallization; Butterworth-Heinemann: Burlington, MA, 2001; pp 358-363. 
${ }^{67}$ Kobyakov, P.S.; Moore, A.; Raguse, J.M.; Swanson, D.E.; Sampath, W.S. Deposition and characterization of $\mathrm{Cd}_{1-\mathrm{x}} \mathrm{Mg}_{\mathrm{x}} \mathrm{Te}$ thin films grown by a novel cosublimation method. $J$. Vac. Sci. Technol. A 2014, 32, 021511-1-021511-7.

${ }^{68}$ Robinson, S.W.; Haynes, D.A.; Rawson, J.M. Co-crystal formation with 1,2,3,5-dithiadiazolyl radicals. CrystEngComm. 2013, 15, 10205-10211.

${ }^{69}$ Qian, H.-F.; Wang, Y.-G.; Geng, J.; Huang, W. A rare case of a dye co-crystal showing better dyeing performance. CrystEngComm. 2015, 17, 2083-2086.

70 Zhang, T.; Yu, Q.; Li, X.; Ma, X. Preparation of 2:1 urea-succinic acid cocrystals by sublimation. J. Cryst. Growth. 2017, 469, 114-118.

${ }^{71}$ Eddleston, M.D.; Sivachelvam, S.; Jones, W. Screening for polymorphs of cocrystals: a case study. CrystEngComm. 2013, 15, 175-181.

72 Shan, N.; Zaworotko, M.J. The role of cocrystals in pharmaceutical science. Drug Discov. Today. 2008, 13, 440-446.

73 Boterashvili, M.; Lahav, M.; Shankar, S.; Facchetti, A.; van der Boom, M.E. On-Surface Solvent-Free Crystal-to-Co-crystal Conversion by Non-Covalent Interactions. J. Am. Chem. Soc. 2014, 136, 11926-11929.

74 Shirman, T.; Freeman, D.; Posner, Y.D.; Feldman, I.; Facchetti, A.; van der Boom, M.E. Assembly of Crystalline Halogen-Bonded Materials by Physical Vapor Deposition. J. Am. Chem. Soc. 2008, 130, 8162-8163.

75 Bailey, R.D.; Buchanan, M.K.; Pennington, W.T. Molecular Complexes of 1,4-Diazines with Iodine. Acta Cryst. 1992, C48, 2259-2262.

76 Vatèle, J.-M. One-Pot Oxidative Conversion of Alcohols into Nitriles by Using a TEMPO/PhI(OAc) $)_{2} / \mathrm{NH}_{4} \mathrm{OAc}$ System. Synlett. 2014, 25, 1275-1278.

77 Scheler, U. High-speed MAS-NMR investigations on radiation-modified fluoropolymers. Solid State Nucl. Magn. Reson. 1998, 12, 9-13.

${ }^{78}$ Bruker. APEX2, 2012, Bruker AXS Inc., Madison, Wisconsin, USA.

79 Altomare, A.; Cascarano, G.; Giacovazzo, C.; Guagliardi, A.; Burla, M.C.; Polidori, G.; Camalli, M. SIR92 - a program for automatic solution of crystal structures by direct methods. J. Appl. Cryst. 1994, 27, 435. 
${ }^{80}$ Sheldrick, G.M. SHELXT - Integrated space-group and crystal-structure determination. Acta Cryst. 2015, $A 71,3-8$.

${ }^{81}$ Farrugia, L. J. WinGX and ORTEP for Windows: an update. J. Appl. Cryst. 2012, 45, 849-854.

${ }^{82}$ Brandenburg, K. Diamond ver. 4.5.1, 2018, Crystal Impact, Bonn, Germany.

${ }^{83}$ Spek, A. L. Structure validation in chemical crystallography. Acta Cryst. 2009, D65, 148-155.

84 Syssa-Magalé, J.-L.; Boubekeur, K.; Palvadeau, P.; Meerschaut, A.; Schöllhorn, B. The tailoring of crystal structures via the self-assembly of organic coordination compounds by $\mathrm{N}$-.I non-covalent halogen bonds: co-crystals of sterically hindered N-heterocycles and 1,4-diiodotetrafluorobenzene. CrystEngComm. 2005, 7, 302-308.

85 Pfrunder, M.C.; Micallef, A.S.; Rintoul, L.; Arnold, D.P.; Davy, K.J.P.; McMurtrie, J. Exploitation of the Menshutkin Reaction for the Controlled Assembly of Halogen Bonded Architectures Incorporating 1,2-Diiodotetrafluorobenzene and 1,3,5Triiodotrifluorobenzene. Cryst. Growth. Des. 2012, 12, 714-724.

${ }^{86}$ Syssa-Magalé, J.-L.; Boubekeur, K., Leroy, J.; Chamoreau, L.-M.; Fave, C.; Schöllhorn, B. Directed synthesis of a halogen-bonded open porphyrin network. CrystEngComm. 2014, 16, 10380-10384.

${ }^{87}$ Pohl, S. Synthese und Kristallstruktur eines polymeren Addukts von 2.2'-Dipyridil mit Iod: $\mathrm{C}_{10} \mathrm{H}_{8} \mathrm{~N}_{2} \mathrm{I}_{6}$. Z. Naturforsch. 1983, 38b, 1535-1538.

${ }^{88}$ Peuronen, A.; Valkonen, A.; Kortelainen, M.; Rissanen, K.; Lahtinen, M. Halogen BondingBased "Catch and Release": Reversible Solid-State Entrapment of Elemental Iodine with Monoalkylated DABCO Salts. Cryst. Growth. Des. 2012, 12, 4157-4169.

${ }^{89}$ Cinčić, D.; Friščić, T.; Jones, W. Structural Equivalence of Br and I Halogen Bonds: A Route to Isostructural Materials with Controllable Properties. Chem. Mater. 2008, 20, 6623-6626.

${ }^{90}$ Bolte, M. CSD Communication, 2004, deposition \# 232562.

91 Cinčić, D.; Friščić, T.; Jones, W. Isostructural Materials Achieved by Using Structurally Equivalent Donors and Acceptors in Halogen-Bonded Cocrystals. Chem. Eur. J. 2008, 14, 747-753.

92 Groom, C.R.; Bruno, I.J.; Lightfoot, M.P.; Ward, S.C. The Cambridge Structural Database. Acta Cryst. 2016, B72, 171-179. 
93 Laurence, C.; Graton, J.; Berthelot, M.; El Ghomari, M.J. The Diiodine Basicity Scale: Toward a General Halogen-Bond Basicity Scale. Chem. Eur. J. 2011, 17, 10431-10444.

${ }^{94}$ Le Questel, Y.-V.; Berthelot, M.; Laurence, C. Hydrogen-bond acceptor properties of nitriles: a combined crystallographic and ab initio theoretical investigation J. Phys. Org. Chem. 2000, 13, 347-358.

${ }^{95}$ Eichele, K. WSolids 1 ver. 1.21.3, 2015, Universität Tübingen.

${ }^{96}$ Amoureux, J.P.; Castelain, M.; Bee, M. An N.M.R. study of molecular motions in the plastic phases of 1-cyanoadamantane. Mol. Phys. 1981, 42, 119-127.

97 Amoureux, J.P.; Decressain, R.; Sahour, M.; Cochon, E. Molecular motions in glassy crystal cyanoadamantane: a proton spin-lattice relaxation study. J. Phys. II France 1992, 2, 249.259 .

98 Sauvajol, J.L.; Lefebvre, J.; Amoureux, J.P.; Bée, M. Dynamical properties of 1cyanoadamantane in the disordered phase. J. Phys. C: Solid State Phys. 1982, 15, 65236532.

99 Lucassen, A.C.B.; Karton, A.; Leitus, G.; Shimon, L.J.W.; Martin, J.M.L.; van der Boom, M.E. Co-Crystallization of Sym-Triiodo-Trifluorobenzene with Bipyridyl Donors: Consistent Formation of Two Instead of Anticipated Three N … I Halogen Bonds. Cryst. Growth Des. 2007, 7, 386-392.

100 Ding, X.-H.; Ou, C.-J.; Wang, S.; Xie, L.-H.; Lin, J.-Y.; Wang, J.-P.; Huang, W. Cocrystallization of 1,3,5-trifluoro-2,4,6-triiodobenzene (1,3,5-TFTIB) with a variety of Lewis bases through halogen-bonding interactions. CrystEngComm. 2017, 19, 5504-5521.

101 Nicolas, I.; Barrière, F.; Jeannin, O.; Fourmigué, M. Sequential Halogen Bonding with Ditopic Donors: $\sigma$-Hole Evolutions upon Halogen Bond Formation. Cryst. Growth Des. 2016, 16, 2963-2971.

102 Roper, L.C.; Präsang, C., Kozhevnikov, V.N.; Whitwood, A.C.; Karadakov, P.B.; Bruce, D.W. Experimental and Theoretical Study of Halogen-Bonded Complexes of DMAP with Di- and Triiodofluorobenzenes. A Complex with a Very Short N $\cdots$ I Halogen Bond. Cryst. Growth Des. 2010, 10, 3710-3720. 
${ }^{103}$ Hidalgo, P.I.; Leal, S.; Jiménez, C.A.; Vöhringer-Martinez, E.; Herrera, B.; Pasán, J.; RuizPérez, C.; Bruce, D.W. Extending the halogen-bonded supramolecular synthon concept to 1,3,4-oxadiazole derivatives. CrystEngComm, 2016, 18, 42-47.

${ }^{104}$ Carletta, A.; Zbačnik, M.; Vitković, M.; Tumanov, N.; Stilinović, V.; Wouters, J.; Cinčić, D. Halogen-bonded cocrystals of $N$-salicylidene Schiff bases and iodoperfluorinated benzenes: hydroxyl oxygen as halogen bond acceptor. CrystEngComm. In press.

105 Aakeröy, C.B.; Wijethunga, T.K.; Benton, J.; Desper, J. Stabilizing volatile liquid chemicals using co-crystallization. Chem. Commun. 2015, 51, 2425-2428.

\section{FOR TABLE OF CONTENTS USE ONLY}

\section{Graphical Abstract}




\section{Synopsis}

Cosublimation is explored and developed as a rapid and simple technique to rapidly produce bulk and single-crystalline samples of a range of halogen-bonded cocrystals. This 'green' solvent-free method enables the production of materials unattainable via other methods. The technique enables broad explorations of polymorphic energy landscapes. 